\title{
Stability analysis and breakup length calculations for steady planar liquid jets
}

\author{
M. R. TURNER ${ }^{1} \uparrow$, J. J. HEALEY ${ }^{2}$, S. S. SAZHIN ${ }^{1}$ \\ AND R. PIAZZESI ${ }^{1}$ \\ ${ }^{1}$ Sir Harry Ricardo Laboratories, School of Computing, Engineering and Mathematics, \\ University of Brighton, Lewes Road, Brighton BN2 4GJ, UK \\ ${ }^{2}$ Department of Mathematics, Keele University, Keele, Staffs ST5 5BG, UK \\ (Received 31 March 2010; revised 9 September 2010; accepted 10 September 2010; \\ first published online 13 December 2010)
}

This study uses spatio-temporal stability analysis to investigate the convective and absolute instability properties of a steady unconfined planar liquid jet. The approach uses a piecewise linear velocity profile with a finite-thickness shear layer at the edge of the jet. This study investigates how properties such as the thickness of the shear layer and the value of the fluid velocity at the interface within the shear layer affect the stability properties of the jet. It is found that the presence of a finite-thickness shear layer can lead to an absolute instability for a range of density ratios, not seen when a simpler plug flow velocity profile is considered. It is also found that the inclusion of surface tension has a stabilizing effect on the convective instability but a destabilizing effect on the absolute instability. The stability results are used to obtain estimates for the breakup length of a planar liquid jet as the jet velocity varies. It is found that reducing the shear layer thickness within the jet causes the breakup length to decrease, while increasing the fluid velocity at the fluid interface within the shear layer causes the breakup length to increase. Combining these two effects into a profile, which evolves realistically with velocity, gives results in which the breakup length increases for small velocities and decreases for larger velocities. This behaviour agrees qualitatively with existing experiments on the breakup length of axisymmetric jets.

Key words: absolute/convective instability

\section{Introduction}

The injection of diesel fuel into an engine cylinder is an important process in the overall running and efficiency of a diesel engine (Hiroyasu, Shimizu \& Arai 1982; Stone 1992; Heywood 1998; Crua 2002). The liquid fuel is injected through an injector (which can have multiple holes), and the resulting jet breaks up into small droplets. These droplets heat up and evaporate, and the mixture of fuel vapour and air burns up in the autoignition and combustion processes. During the injection process a useful quantity to predict, or measure, is the breakup length of the jet. This is the length over which the jet remains intact before it begins to break up into ligaments and droplets. Modelling this injection process is an important integral part of the computational fluid dynamics (CFD) diesel engine models (Sazhin et al. 2003, 2008). 
During the injection process these jets undergo acceleration, but like most current injection models, we neglect this process, and in this paper we give a more complete analysis of the steady-jet problem by studying parameter ranges that cover those seen in diesel injection models, as well as extending the research to look at parameters that cover a wider range of jets which will be of interest to problems outside of diesel injection problems. Furthermore, when the jet acceleration is relatively weak, the jet may be treated using a quasi-steady approximation which can give some qualitative insight into how acceleration may affect breakup. Thus this analysis is expected to help with future studies of the unsteady-jet problem. While the jets in diesel engines are close to axisymmetric jets, in this paper we consider only planar jets, as these allow us to greatly simplify the analysis and lead to analytical results. The stability and breakup properties of both axisymmetric and planar jets have been studied both experimentally and theoretically in the past, and for a good overview of these studies the reader is directed to the introduction of Söderberg \& Alfredsson (1998).

A planar jet consists of two parallel shear layers where the vorticity at each layer is equal and opposite. The stability properties of such jets are found by performing a linear stability analysis about a basic velocity profile $U(y)$, where $y$ is a coordinate normal to the jet axis. By looking for a travelling-wave solution of the linearized Navier-Stokes equations of the form $\hat{v}(x, y, t)=v(y) \exp [\mathrm{i}(\alpha x-\omega t)]$, in the absence of viscosity, we arrive at the Rayleigh equation

$$
(\alpha U-\omega)\left(\frac{\mathrm{d}^{2} v}{\mathrm{~d} y^{2}}-\alpha^{2} v\right)-\alpha \frac{\mathrm{d}^{2} U}{\mathrm{~d} y^{2}} v=0,
$$

where $v(y)$ is the velocity component normal to the jet axis (in the $y$-direction); $t$ is time; $\alpha$ is the streamwise wavenumber; and $\omega$ is the angular frequency (see Drazin \& Reid 1981). Here we neglect the effect of viscosity, as typical Reynolds numbers for diesel jets are $O\left(10^{4}\right)$ or larger, which is larger than $10^{3}$, a value above which viscosity can be neglected in channel flows (Rees \& Juniper 2010). Using a piecewise linear profile for $U(y)$ allows an analytic form of the dispersion relation $D(\alpha, \omega)=0$ to be derived, on which a temporal stability analysis can be performed. The dispersion relation can be solved for complex $\omega$, for a given real $\alpha$. For the case of planar and axisymmetric jets, the range of real wavenumbers which exhibit growth is governed by the width of the shear layer and the magnitude of the surface tension (Rayleigh 1894; Batchelor \& Gill 1962; Funada, Joseph \& Yamashita 2004; Marmottant \& Villermaux 2004). However, a temporal stability analysis does not show certain aspects of the jet stability, such as whether or not it is absolutely or convectively unstable, which has important implications for where it breaks up. The answer to this question requires a different mathematical approach.

This approach uses spatio-temporal stability analysis (Heurre \& Monkewitz 1990) in which both $\alpha$ and $\omega$ are allowed to become complex, and growth rates are obtained in various frames of reference moving in the axial direction along the jet. The growth rate is calculated using the method of steepest descent (Hinch 1991) by searching for special saddle points in the complex $\alpha$-plane through which the inverse-Fouriertransform contour can be deformed (see $\S 2.1$ ). The saddle point on the contour with the largest growth rate gives the disturbance growth rate in the limit $t \rightarrow \infty$. For a liquid jet there is one saddle point in the $\alpha$-plane whose position is determined by the thickness of the shear layers and the value of the surface tension (known as the ' $s_{1}$ ' saddle in Juniper 2007 and 'shear layer mode' in Lesshafft \& Huerre 2007), and this saddle is located close to the real $\alpha$-axis. Also in the $\alpha$-plane is a set of saddle points 
close to the imaginary $\alpha$-axis which are due to the interaction between the two shear layers (known as ' $s_{2}$ ' saddles in Juniper 2007 and 'jet column modes' in Lesshafft \& Huerre 2007). In the present study we examine how the growth rate at these saddles and their positions are affected when the interface between the two fluids is placed within the shear layer. We find that this leads to important qualitative differences compared with previous studies in which the density interface was assumed to lie on only one side of the shear layer (Marmottant \& Villermaux 2004; Juniper 2007). Our assumption to neglect viscosity in this study is valid because $\mathrm{Yu} \&$ Monkewitz (1990) showed that the transition to absolute instability is caused by the interaction between the two shear layers and is not a viscous effect. Lin, Lian \& Creighton (1990) and Li \& Tankin (1991) investigated the effect of viscosity on planar liquid jets and found that the solution contained an unstable mode with zero frequency and two convectively unstable modes, identical to those of Hagerty \& Shea (1955), whose growth rates are affected by the presence of viscosity. Typically viscosity has a damping effect on the instabilities, but in particular parameter regimes viscosity enhances one of the convective modes ( $\mathrm{Li} \&$ Tankin 1991).

The parameters we wish to investigate in this study are as follows: $q=\rho_{2} / \rho_{1}$, the density ratio of the outer fluid to that of the liquid jet; $W=W e^{-1}$, the inverse Weber number (defined below); $\delta_{1}$ and $\delta_{2}$, the thicknesses of the shear layers on either side of the fluid interface; and the ratio of the fluid velocity at the interface to the maximum jet velocity $\beta$. It is known that the density ratio has a large effect on the behaviour of absolute instabilities, in particular that low-density jets $(q>1)$ are almost always absolutely unstable (Sreenivasan, Raghu \& Kyle 1989; Yu \& Monkewitz 1990; Juniper 2006). In this work we are concerned with jets which have $q<1$, but we find that absolute instabilities can occur at these density ratios for particular velocity profiles. Values of $q<1 / 10$ can typically be found in diesel jet injection experiments as the pressure of the gas inside the cylinder is varied, while values of $q>1 / 10$ provide a wider range of interest to the reader. Rees \& Juniper (2009) showed that the effect of small and moderate surface tension values is to increase the magnitude of any absolute instability that arises due to the varicose modes of low-density jets, while larger surface tension values are ultimately a stabilizing feature of the flow. This is one of the main differences between planar jets and axisymmetric jets, where surface tension has a more destabilizing effect. The effect of the shear layers in both fluids, and the magnitude of the fluid velocity at the fluid interface, on the absolute instability properties of jets with $q<1$ has not been explored to date and will form part of this investigation. The case of low-density jets $(q>1)$ with a fluid interface in the shear layer has been examined using smooth velocity and density profiles (Raynal et al. 1996; Srinivasan, Hallberg \& Strykowski 2010). These studies show that low-density jets experience a transition from absolute to convective instability if the shear layer thickness is sufficiently large compared with the jet diameter.

The stability results are then used to estimate breakup lengths of steady jets, which are compared with experiments such as those of Hiroyasu, Shimizu \& Arai (1982). The experiments show that breakup lengths increase with injection velocity for small injection velocities and then reduce for larger velocities before eventually levelling off (see figure 13 of Hiroyasu et al. 1982 which is reproduced as figure 1 here). In the comprehensive review paper by Eggers \& Villermaux (2008) the reducing breakup length for larger velocities is explained by the thinning shear velocity at the edge of the jet, but no explanation is given for the rise in the breakup length for small velocities. In this paper we show that the increasing breakup length for small velocities could be due to the value of the fluid velocity at the fluid interface within the shear layer 


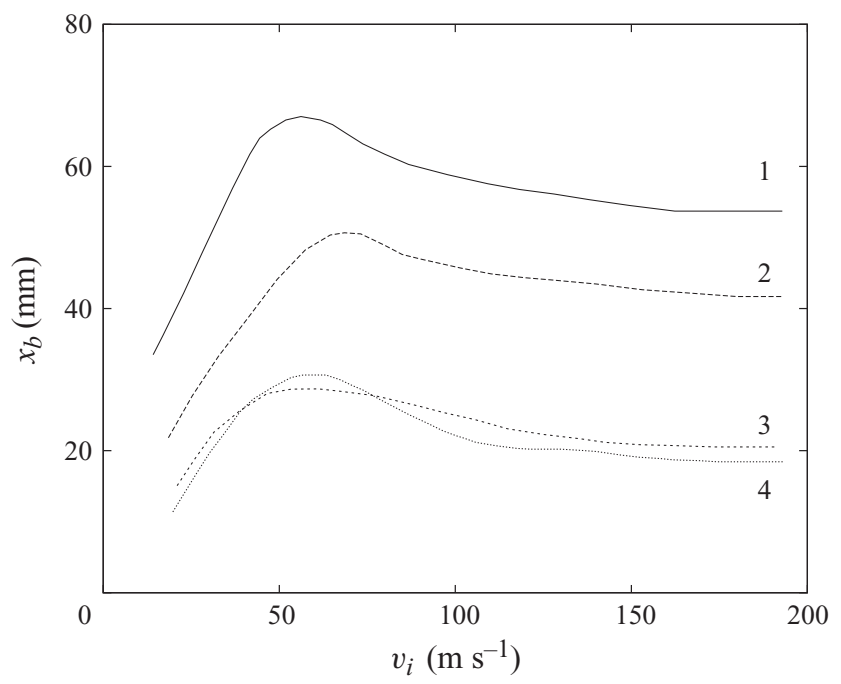

FiguRE 1. Plot of the breakup length $x_{b}(\mathrm{~mm})$ as a function of the injection velocity $v_{i}$ $\left(\mathrm{m} \mathrm{s}^{-1}\right)$, for an experiment in which water is injected into pressurized nitrogen. The results (from Hiroyasu et al. 1982) are for four different nitrogen pressures, which correspond to $q \approx 1 / 1000,1 / 100,1 / 30$ and $1 / 20$. These are numbered $1-4$ respectively. The radius of the nozzle used in this experiment was $0.15 \mathrm{~mm}$.

increasing with velocity. The experiments of Hiroyasu et al. (1982) were not performed using diesel jets, but this experiment has non-dimensional parameters which coincide with those we expect in diesel injection systems.

The present paper is laid out as follows. In $\S 2$ we formulate the problem and derive the analytic dispersion relation which determines the stability characteristics of the jet. Then follows a brief discussion of the spatio-temporal stability method used to analyse the jet stability, including, in $\S 2.2$, a discussion of the $s_{1}$ and $s_{2}$ saddle points in the complex $\alpha$-plane and how they move around as the problem parameters vary. In $\S 3$ we calculate the growth rates for particular parameter regimes and investigate the appearance or otherwise of absolute instabilities. In $\S 4$ we use the convective instability analysis to examine the breakup length of a steady liquid jet and compare the results with those in figure 1 . Our concluding remarks and discussion can be found in $\S 5$.

\section{Formulation of the mathematical model}

We consider a two-dimensional steady planar jet orientated along the $x^{*}$-axis in the $\left(x^{*}, y^{*}\right)$-plane with dimensional reference velocity $U_{0}^{*}$ at $y^{*}=0$, emerging from a nozzle of thickness $2 L^{*}$ at $x^{*}=0$. Using this reference length and velocity we can define dimensionless variables such as its velocity $U\left(=\right.$ velocity of the jet $\left./ U_{0}^{*}\right)$ and its thickness $2 L$ (where $L=$ thickness of the jet $/ 2 L^{*}$ ). The jet fluid has density $\rho_{1}$ and lies between an outer fluid of density $\rho_{2}$. For the stability analysis in $\S 3$ we consider a jet profile with $\max (U) \equiv V=1$; however when we consider breakup lengths for these jets in $\S 4$, we will allow $V$ to vary. Thus we leave $V$ explicitly in the equations in this section for completeness.

We assume that the jet does not spread significantly as we move along the $x$-axis in the region we wish to consider, and we neglect any streamwise variation of the jet in this paper. Therefore we can consider the basic velocity profile $\boldsymbol{u}=U(y) \boldsymbol{i}$ as a 
(a)

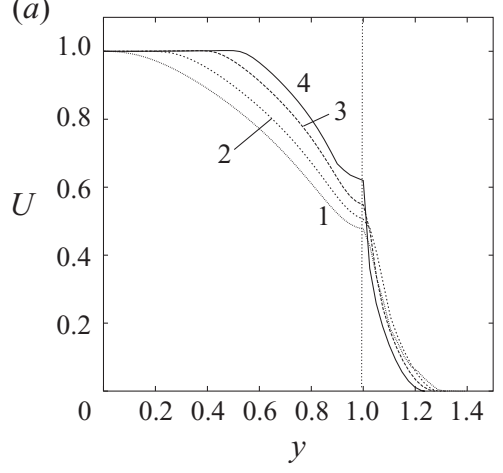

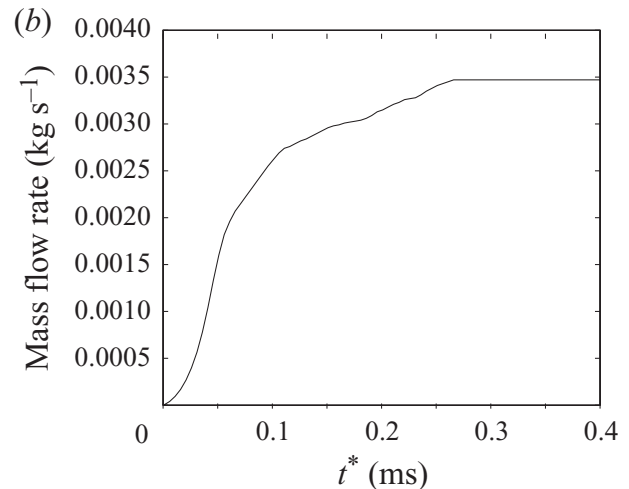

Figure 2. (a) Plot of the normalized velocity profiles, taken at $0.1 \mathrm{~mm}$ from the nozzle exit, for the CFD simulation with $q \approx 1 / 45$ for an axisymmetric jet with the dimensional maximum velocity of $44,81,182$ and $340 \mathrm{~m} \mathrm{~s}^{-1}$, numbered $1-4$ respectively. The fluid interface is denoted by the vertical dotted line. ( $b$ ) The experimental mass flow rate used to generate result 4 of $(a)$. Note that the mass flow rate has been modified to enter a steady state for $t^{*}>2.5 \times 10^{-4} \mathrm{~s}$. In this simulation $\rho_{\text {fuel }}=850 \mathrm{~kg} \mathrm{~m}^{-3}$; the pressure inside the cylinder is $2 \mathrm{MPa}$, and the initial temperature of the fuel and air is $355 \mathrm{~K}$.

function of the normal coordinate $y$ only, where $i$ is the unit vector in the streamwise direction. This assumption is valid close to the nozzle, where the jet spreads slowly in space. Typically this velocity profile will be smooth with shear layers in each fluid at the edge of the jet, such as in the CFD simulations in figure 2 . The velocity profiles in figure 2(a) are calculated for an axisymmetric diesel jet being injected in static air, normalized by their axial velocity along the centre of the jet and non-dimensionalized by the radius of the nozzle $\left(L^{*}=0.0675 \mathrm{~mm}\right)$. These profiles are generated using the CFD package ANSYS FLUENT, where the boundary condition for the mass flow rate of fluid in the nozzle is given by measurements taken from an in-house experiment (see figure 2b) (Karimi 2007). The plotted profiles are taken at $t^{*}=3 \times 10^{-4} \mathrm{~s}$ where the jet has reached a steady state.

The velocity profiles in figure 2 are calculated using the Eulerian multi-phase model. In this model a momentum equation for each fluid phase is solved for, giving the respective velocity field. Since there exist large velocity differences between the two phases, this approach allows us to overcome the limitations of the shared velocity and temperature formulation of the volume-of-fluid model, which can affect the fluid velocities computed across the interface. We consider the two fluids to be immiscible, and the geo-reconstruction sharpening scheme (Youngs 1982; Ferziger \& Peric 2004) is used to construct the free surface. The computational domain is a closed cylinder $80 \mathrm{~mm}$ in length and $25 \mathrm{~mm}$ in the radial direction, which was chosen to approximate the cylinder of an engine in the experimental facilities at the University of Brighton. The nozzle is approximated by a cylindrical channel of $1.08 \mathrm{~mm} \times 0.135 \mathrm{~mm}$ (axial direction $\times$ radial direction) and is located at the centre of the main cylinder edge. The computational domain is covered by a structured mesh of approximately 82000 nodes which is refined inside the nozzle and in a $0.5 \mathrm{~mm} \times 0.3 \mathrm{~mm}$ region immediately outside the nozzle. A coarser and unstructured mesh is used outside this region, and a time step of $\Delta t^{*}=5 \times 10^{-8} \mathrm{~s}$ is used. A standard $\kappa-\epsilon$ turbulent model for both fluids is used. Initially the air in the chamber is considered at rest with a temperature of $355 \mathrm{~K}$ and a pressure of $2 \mathrm{MPa}$. The fuel is injected into the cylinder through the nozzle at the constant temperature of $355 \mathrm{~K}$, assuming an adiabatic condition on the 


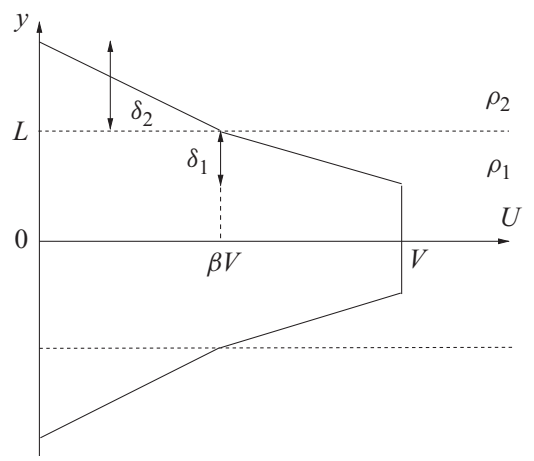

Figure 3. Plot of the piecewise velocity profile $U(y)$, where the thickness of the liquid jet is $2 L$. The density of the liquid layer is $\rho_{1}$ and has a shear layer width of $\delta_{1}$, while the air has density $\rho_{2}$ and a shear layer thickness $\delta_{2}$. The parameter $\beta \in[0,1]$ defines the jet velocity at the fluid interface, normalized by $V$.

walls and applying a mass flow rate boundary condition, given by figure $2(b)$, at the nozzle inlet surface. This produces a non-uniform velocity profile as the fuel enters the main cylinder. A check of the dependency of the results on the numerical grid was also carried out, and the results were found to agree within a few per cent; hence the simulations are consistent.

The mass flow rate in figure $2(b)$ has been modified so that it levels off once the initial acceleration of the jet has been completed at around $t^{*}=2.5 \times 10^{-4} \mathrm{~s}$. Beyond this time the jet reaches a steady state, although from $t^{*}=2.5 \times 10^{-4} \mathrm{~s}$ onwards, the change in the profiles is very small. The other profiles are generated by considering fractional multiples of this mass flow rate to generate the lower-velocity jets. These profiles are generated assuming that the jet is axisymmetric, but we expect qualitatively similar results for a planar jet, so we use these results to motivate the velocity profiles used in this study. In fact the experimental results of Söderberg \& Alfredsson (1998) show planar jet velocity profiles which have a similar appearance to those shown here; however they cannot determine the structure of the velocity profile in the outer fluid as we can in our CFD simulations. The profiles in figure 2(a) are taken at $0.1 \mathrm{~mm}$ from the nozzle exit, and the nozzle is assumed to be full of fluid for all times to best model the flow in a diesel injector where the nozzle fills with fluid as the injector needle is lifted. In this study we approximate the CFD profiles in figure 2 by a piecewise linear velocity profile, which is shown in figure 3 . Although this profile is a simplification of the true profile, it captures important qualitative aspects of the jet and greatly simplifies the problem when it comes to studying properties such as absolute instabilities. This piecewise linear profile exhibits the same qualitative behaviour as a realistic smooth profile, as the exact shape of the shear layer has only a small effect on the stability characteristics of the flow (Esch 1957); further, Healey (2009) found a co-flow absolute instability for certain confined piecewise linear shear layers and the same qualitative behaviour, with modest quantitative variation, in smooth profiles. The piecewise linear profile also allows for an analytical expression for the dispersion relation, as well as implicitly capturing typical features of a viscous jet profile, although we do not explicitly consider the effects of viscosity in this model. Other studies have considered the stability of planar jets, using velocity profiles which are more realistic, i.e. have shear layers on either side of the fluid interface, than the simple plug flow approximation (Hashimoto \& Suzuki 1991; Söderberg \& Alfredsson 
1998; Söderberg 2003), but the current paper is the first to examine the stability properties of such a realistic profile through the spatio-temporal stability analysis approach.

The piecewise linear velocity profile in figure 3 is symmetric about $y=0$; hence we need only to consider half the jet, of which the top half has the form

$$
U(y)= \begin{cases}0, & y>L+\delta_{2}, \\ -\frac{\beta V}{\delta_{2}}\left(y-L-\delta_{2}\right), & L+\delta_{2}>y>L, \\ V-\frac{(1-\beta) V}{\delta_{1}}\left(y-L+\delta_{1}\right), & L>y>L-\delta_{1}, \\ V, & L-\delta_{1}>y>0 .\end{cases}
$$

The parameter $\beta$ defines the jet velocity at the fluid interface normalized by the velocity $V$. It can be seen in figure 2 that $\beta$ increases with increasing $V$ by considering the velocity at the fluid interface. In figure 3 we number the layers of the profile 1-4 from the top layer in fluid 2 to the centre layer in fluid 1 . The value of $L$ in this study will be set to $L=1$; i.e the thickness of the jet is the same as that of the nozzle, although a true value of $L$ will be slightly smaller than unity because of a thinning of the jet as it leaves the nozzle (Domann \& Hardalupas 2004). This effect is expected to be relatively small in the region of interest, so we neglect it here.

The stability of profile (2.1) to linear disturbances, in the absence of viscosity, is found by linearizing the two-dimensional Euler equations. We introduce velocity and pressure fluctuations of the form

$$
(\hat{u}, \hat{v}, \hat{p})(x, y, t)=(U(y), 0,0)+\epsilon(u(y), v(y), p(y)) \mathrm{e}^{\mathrm{i}(\alpha x-\omega t)}+\text { c.c. },
$$

into the Euler equations, where $\epsilon \ll 1$ and $u, v, p=O(1)$, and time has been nondimensionalized by $L^{*} / U_{0}^{*}$. By neglecting nonlinear terms and eliminating the pressure $p$ and the streamwise velocity perturbation $u$ we arrive at the Rayleigh equation (1.1) in each of the fluid layers (Drazin \& Reid 1981). Here $\alpha$ is the wavenumber in the streamwise direction and $\omega$ is the angular frequency of the disturbance, such that $\omega / \alpha=c$ is the wave phase speed in the $x$-direction. For high-speed jets, it is likely that the fluid within the jet is close to or could even be turbulent, possibly owing to the cavitation in the nozzle (Arcoumanis et al. 2001). However in this study we assume that any eddies in the jet are small, and therefore our assumption that the jet appears as a single velocity profile and can be approximated as (2.1) still holds.

The modal solutions to the Rayleigh equation can be either sinuous (even functions for $v ; v(y=0)=1, \mathrm{~d} v / \mathrm{d} y(0)=0$ ) or varicose (odd functions for $v ; v(0)=0$, $\mathrm{d} v / \mathrm{d} y(0)=1)$ modes, and as any perturbation can be made up of a linear combination of these modes we have a complete stability representation by considering these modes only. Therefore for the piecewise linear basic profile (2.1), the form of the eigenmodes can be solved for exactly in each layer. Also, by using the symmetry conditions at $y=0$ and the two matching conditions

$$
\Delta\left[\frac{v_{j}}{\alpha U_{j}-\omega}\right]=0, \quad \Delta\left[\rho_{j}\left(\alpha U_{j}-\omega\right) v_{j}^{\prime}-\rho_{j} \alpha U_{j}^{\prime} v_{j}\right]=\chi,
$$

across each velocity layer to eliminate the arbitrary constants of the problem, we can derive the dispersion relation

$$
D(\alpha, \omega)=\hat{c}_{4} \omega^{4}+\hat{c}_{3} \omega^{3}+\hat{c}_{2} \omega^{2}+\hat{c}_{1} \omega+\hat{c}_{0}=0,
$$


where $\hat{c}_{4}$ to $\hat{c}_{0}$ are functions of $\left(\alpha, \delta_{1}, \delta_{2}, \beta, V, L, q=\rho_{2} / \rho_{1}, W\right)$ given in the Appendix. Here the notation is $\Delta[]=[]_{y_{0}-\epsilon}^{y_{0}+\epsilon}$ at the discontinuity $y=y_{0}$ and $\epsilon \rightarrow 0$. When the second interface condition is applied between two layers of the same fluid, $\chi=0$; however, $\chi=W \alpha^{4} \rho_{1}$ at the interface between the two fluids at $y=L(=1)$. The non-dimensional constant $W=W e^{-1}=\sigma /\left(\rho_{1} U_{0}^{* 2} L^{*}\right)$, where $\sigma$ is the dimensional surface tension, is the inverse of the Weber number. In the present study this parameter remains constant, but the surface tension effects appear as $W / V^{2}$ in the above dispersion relation, so as $V$ increases, the effect of surface tension reduces. Consequently we could fix $V=1$ and let $W$ vary, but in $\S 4$ we calculate the breakup length of the jet as a function of $V$.

For the case of no surface tension, $W=0$, the dispersion relation reduces to

$$
c_{3} \omega^{3}+c_{2} \omega^{2}+c_{1} \omega+c_{0}=0,
$$

by division of the factor $(1 / 2 \alpha V-\omega)$. The expressions for $c_{3}$ to $c_{0}$ can also be found in the Appendix. For more information of the derivation of the dispersion relation, see Drazin \& Reid (1981), Schmid \& Henningson (2001), Healey (2007) or Juniper (2007).

\subsection{Spatio-temporal stability analysis}

In this study we are interested in how disturbances generated at the nozzle propagate along the jet. Thus it appears that a simple temporal instability analysis for each real wavenumber $\alpha$ would suffice for finding unstable waves. However this analysis does not allow for distinguishing an absolute instability from a convective one, i.e. distinguishing a disturbance which grows at the same spatial position at which it was forced (the nozzle in this case) from one which grows only as it propagates downstream. In a steady parallel jet, an absolute instability is significant because the jet will eventually break up at the nozzle as long as enough time is allowed to pass. In this paper we examine the fluid response to the forcing in frames of reference moving at various speeds downstream (or possibly upstream) of the source of disturbances as in the problems considered by Healey (2006) and Juniper (2006).

The calculation of this response in one spatial dimension can be found in works such as Huerre (2000) and Healey (2006) and is outlined below. We assume that a time-dependent forcing is turned on at $t=0$ at the nozzle of the jet $(x=0)$ and that this can be written as the boundary condition $\hat{v}(x, 0, t)=\delta(x) \hat{f}(t)$, where $\hat{f}=0$ for $t<0$ (Juniper 2007). The solution for $\hat{v}(x, y, t)$ can be written as the double-inverse Fourier transform

$$
\hat{v}(x, y, t)=\frac{1}{4 \pi^{2}} \int_{F_{\alpha}} \int_{L_{\omega}} \frac{f(\omega)}{D(\alpha, \omega)} v(y ; \alpha, \omega) \mathrm{e}^{\mathrm{i}(\alpha x-\omega t)} \mathrm{d} \alpha \mathrm{d} \omega,
$$

where $D(\alpha, \omega)$ is the dispersion relation (2.4). The integration contour $F_{\alpha}$ runs from $-\infty$ to $\infty$ along the real axis in the complex $\alpha$-plane, while the contour $L_{\omega}$ runs from $-\infty$ to $\infty$ above all singularities in the complex $\omega$-plane to ensure $\hat{v}=0$ for $t<0$.

It is possible to distinguish between convective and absolute instabilities without numerically evaluating the above double integral (Briggs 1964), by considering an impulsive forcing $\hat{f}(t)=\delta(t)$. The residue theorem can then be used to evaluate the $\omega$ integration in (2.6) as

$$
\hat{v}(x, y, t)=-\frac{\mathrm{i}}{2 \pi} \sum_{m} \int_{F_{\alpha}} \frac{v(y ; \alpha, \omega)}{D_{\omega}\left(\alpha, \omega_{m}(\alpha)\right)} \mathrm{e}^{\mathrm{i}\left(\alpha x-\omega_{m} t\right)} \mathrm{d} \alpha,
$$


where $\omega_{m}$ is the $m$ th root of the dispersion relation. Equation (2.7) can now be evaluated by the method of steepest descent (Hinch 1991), where in the large time limit with $x / t=O(1)$ the dominant contribution to the integral comes from the particular saddle points of the function

$$
g_{m}=\omega_{m}-\alpha \frac{x}{t},
$$

where

$$
\frac{\partial \omega_{m}}{\partial \alpha}=\frac{x}{t} .
$$

In this study we consider the growth of the perturbation $\hat{v}$ along all possible real characteristics $\partial \omega / \partial \alpha=x / t$ and calculate the growth rate

$$
g_{i}=\operatorname{Im}(g)=\omega_{i}-\frac{x}{t} \alpha_{i},
$$

where the subscript $i$ denotes the imaginary part and we have dropped the subscript $m$ for clarity, knowing that we must consider all Riemann surfaces. The saddle points of the dispersion relation are found by simultaneously solving equations $\bar{D}(g, \alpha)=\bar{D}_{\alpha}(g, \alpha)=0$, numerically using Newton iterations, where $\bar{D}(g, \alpha)$ is given by (2.4) with $\omega$ replaced by (2.8).

\subsection{Distinction between saddle points}

In this section we show how the integral in (2.7) is evaluated, consider the two types of saddle points which contribute to the integral and show how the saddles move around the $\alpha$-plane as we consider the growth along various $x / t$ characteristics.

The integration path in (2.7) originally lies along the real axis in the complex $\alpha$ plane. However the method of steepest descent makes the evaluation of this contour easier by deforming the integration path to pass through particular saddle points in the $\alpha$-plane. This deformation of the integration contour can only be carried out as long as no poles or branch cuts of $g(\alpha)$ (which correspond to those of $\omega(\alpha)$ ) are crossed. We visualize the integration by plotting contours of $g_{i}$ in the complex $\alpha$-plane and then choose an integration path which solely lies within the valleys of the saddle point (Healey 2006, 2007). A sensible choice for the inversion contour is a path which follows the contours of the constant $\operatorname{Re}(g)=g_{r}$ (orthogonal to the contours of $\left.g_{i}\right)$ which is allowed to change values of $g_{r}$ only at points in the $\alpha$-plane where $g_{i}$ is strongly negative. This is done so as to only add a negligible contribution to the integral if we were to evaluate it numerically. If more than one saddle point with $g_{i}>0$ lies on the integration path, then both have to be considered for the growth rate of the disturbance. However the long-time response can solely be inferred from the values of the saddle with the largest value of $g_{i}$, henceforth known as the dominant saddle point.

An example of what the contours of $g_{i}$ look like in the complex $\alpha$-plane for the sinuous mode with $\left(\delta_{1}, \delta_{2}, q, \beta, V, W\right)=(0.5,0.5,1 / 500,1 / 2,1,0)$ and $x / t=0.6$ and $x / t=0.9$ can be seen in figures $4(a)$ and $4(b)$ respectively. These parameters are chosen to give a typical velocity profile with non-zero shear layers in each fluid. The density ratio $q=1 / 500$ is considered because it corresponds to cold liquid diesel liquid fuel being injected into compressed air at about 5 atmospheres pressure, which is similar to the smallest density ratio in the experiments in figure 1. In this paper we adopt the notation of Juniper $(2006,2007)$ for the labelling of the saddle points. We denote the saddle whose position is controlled by the thickness of the shear layer and surface tension (Rees \& Juniper 2009) as $s_{1}$. This saddle corresponds to 

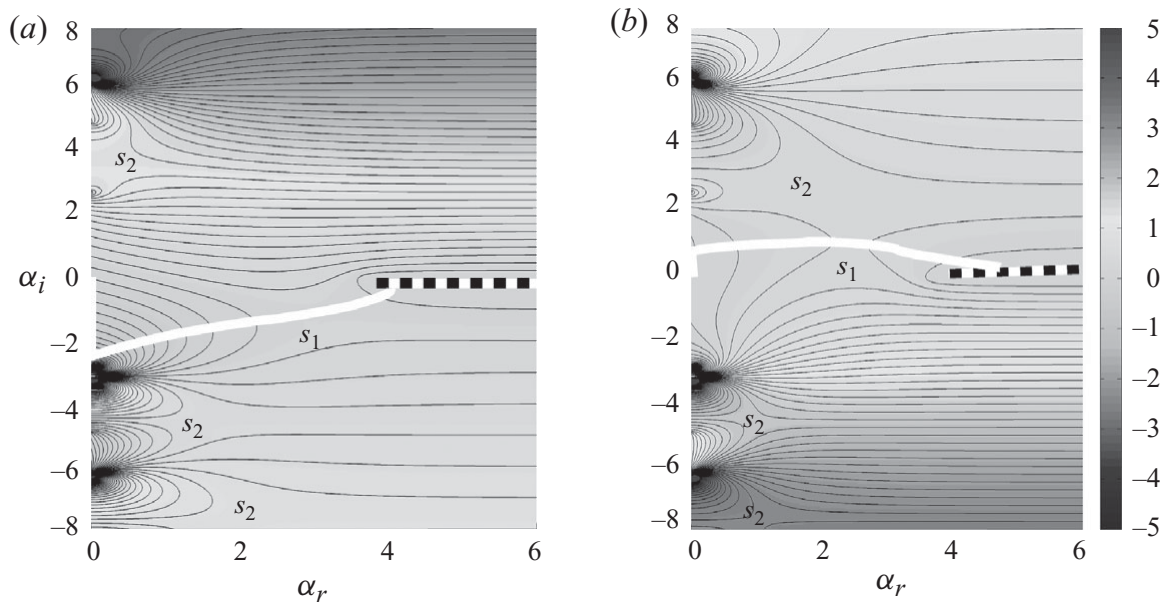

Figure 4. Plot of the contours of $g_{i}$ in the complex $\alpha$-plane for the sinuous mode with $\left(\delta_{1}, \delta_{2}, q, \beta, V, W\right)=(0.5,0.5,1 / 500,1 / 2,1,0)$ and $(a) x / t=0.6$ and $(b) x / t=0.9$. The branch cut near the real axis is marked by the dashed line, while the path of integration is shown by the solid white line. The saddles are labelled $s_{1}$ and $s_{2}$ as in Juniper (2007).

waves with moderate and short wavelengths so that the eigenfunctions are confined to a region close to the shear layer, so shear layer effects and surface tension are important for determining its position. The other saddles which are controlled by the interaction of the two shear layers (Juniper 2007) are denoted as $s_{2}$ saddles. Unlike the $s_{1}$ saddles, these $s_{2}$ saddles correspond to waves with long wavelengths which have wide eigenfunctions that feel the effect of both shear layers. As well as the branch cut close to the real axis (denoted by the dashed line) there are branch cuts close to the imaginary $\alpha$-axis, which we have not shown for clarity. These branch cuts lie so close to the imaginary $\alpha$-axis, such as the one which lies approximately between the origin and $\alpha_{i}=-3$, that they do not affect our choice of inversion contour in this study. The position of all the branch points in the $\alpha$-plane can be found by simultaneously solving equations $\bar{D}(g, \alpha)=\bar{D}_{\omega}(g, \alpha)=0$, using Newton iterations. For both cases in figure 4 we note that the only saddle which lies on the contour of integration is the $s_{1}$ saddle. For $x / t=0.6$ in figure $4(a)$ we see that the $s_{1}$ saddle lies below the real $\alpha$-axis, so the inversion contour comes between the two branch points at the origin, down the right-hand side of the branch cut close to the imaginary axis, and then passes over the $s_{1}$ saddle. The contour then passes around the branch cut close to the real $\alpha$-axis and off to infinity above the branch cut, but this is not shown here. As $x / t \rightarrow 0.5$ from above, the $s_{1}$ saddle remains the only saddle point on the integration contour, and it moves to large $\alpha_{r}$ while the magnitude of $g_{i}$ tends to zero. As $x / t$ increases from 0.6 the $s_{1}$ saddle moves around the branch cut on the real $\alpha$-axis and on to a Riemann sheet with $\alpha_{i}>0$, which can be seen for $x / t=0.9$ in figure $4(b)$. In this case the dominant $s_{1}$ saddle is very close to an $s_{2}$ saddle in the upper complex plane, but an investigation of the valleys of the respective saddle points shows that for this parameter set the inversion contour cannot pass over both saddle points. Therefore as $x / t \rightarrow 1$ the $s_{1}$ saddle remains dominant and again moves to large $\alpha_{r}$, with $g_{i} \rightarrow 0$, but this time above the branch cut near the real axis.

As the density ratio $q$ is increased, the $s_{1}$ saddle moves closer to the imaginary $\alpha$-axis, but in this paper we find that the $s_{2}$ saddles do not contribute to the stability of the jet. However if the jet were confined between two solid surfaces, then a further 


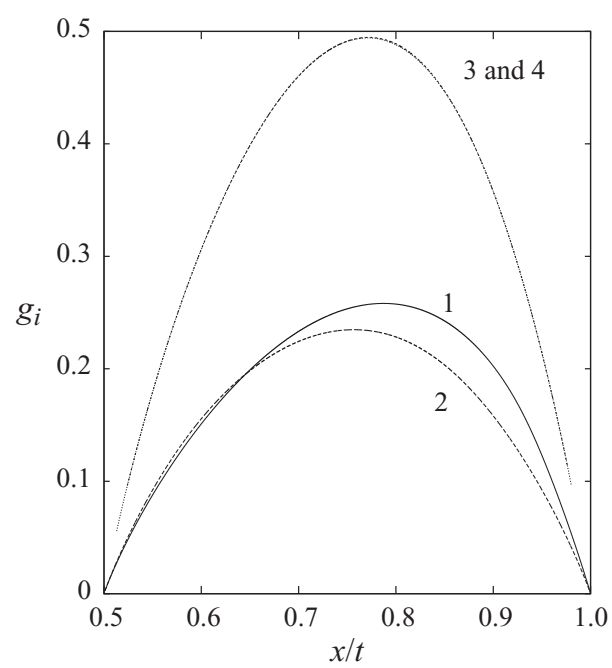

FIGURE 5. Plot of the growth rate $g_{i}$ as a function of $x / t$ for the sinuous mode with $\left(\delta_{1}, \delta_{2}, q, W\right)=(0.5,0.5,1 / 500,0)$, the varicose mode with $(0.5,0.5,1 / 500,0)$, the sinuous mode with $(0.25,0.25,1 / 500,0)$ and the varicose mode with $(0.25,0.25,1 / 500,0)$, numbered $1-4$ respectively.

set of $s_{2}$ saddles would be introduced to the $\alpha$-plane, and then as the position of the walls is varied, these $s_{2}$ saddles could become traversed by the integration contour and hence affect the stability properties of the jet as found in Juniper (2007). This, however, is not considered in the present paper.

We note that an absolute instability will occur in the jet if there exists a saddle point on the inversion contour with $g_{i}>0$ for $x / t=0$. These absolute instabilities are significant for steady jets, because eventually the flow will break down at the nozzle as long as we allow enough time to pass. Thus, determining their existence for particular parameter values is very important, and in the next section we calculate parameter sets with absolute instabilities that have not been documented before.

\section{Stability calculations for a steady jet}

In this section we present a systematic study of the convective and absolute stability properties of the velocity profile (2.1) for a wide range of parameter values, such as the shear layer thickness, surface tension and the value of the jet velocity at the fluid interface. In $\S 4$ we use the relevant results which relate to our profiles in figure $2(a)$ to make a connection with jet breakup lengths. Throughout this section we set $V=L=1$ without affecting the qualitative nature of the results, and unless otherwise stated $\beta=1 / 2$.

In figure 5 we plot the growth rate $g_{i}$ as a function of the characteristic $x / t$ for both sinuous (results 1 and 3) and varicose (results 2 and 4) modes. For both parameter sets the varicose mode has a smaller maximum growth rate value $g_{i}^{\max }$, where $g_{i}^{\max }$ is the maximum value of $g_{i}$, although as the shear layers thin out, the growth rates for both the sinuous and varicose modes tend to the same values, as is shown by results 3 and 4 which are indistinguishable from one another. This is because as the shear layer thins, the wavelengths shorten so that the eigenfunctions decay faster with distance from the shear layer, and therefore, whether $v(0)=0$ or $v^{\prime}(0)=0$ is used makes very little difference. In this limit the results should approach those of an isolated mixing 

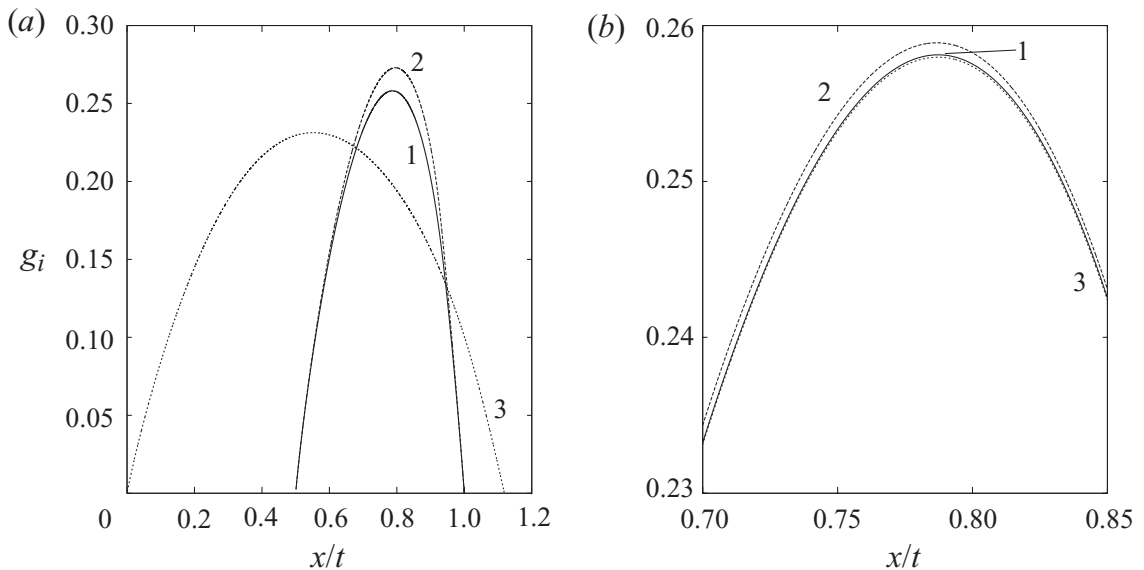

Figure 6. (a) Plot of the growth rate $g_{i}$ for sinuous modes as a function of $x / t$ for $(0.5,0.5,1 / 500,0),(0.5,0.5,1 / 10,0)$ and $(0.5,0.5,1,0)$ numbered $1-3$ respectively. $(b)$ The sinuous mode growth rates for $(0.5,0.5,1 / 500,0),(0.5,0.1,1 / 500,0)$ and $(0.5,0.9,1 / 500,0)$ numbered $1-3$ respectively.

layer, as studied in Healey (2009), but with a modification due to the fluid interface being placed in the middle of the shear layer. In fact, the varicose modes correspond to the case of a mixing layer confined by a single plate studied in Healey (2009). As the sinuous modes have larger maximum growth rate, these modes will become unstable first and will therefore be significant to convective instabilities if we are interested in the initial breakup of the jet. However the varicose modes also need to be considered in case they produce a shorter breakup length. The varicose modes are most significant for the absolute instabilities in the jet, and it should be noted that the varicose mode with $x / t \lesssim 0.64$ and $\delta_{1}=\delta_{2}=0.5$ has a growth rate larger than the sinuous modes as well as a maximum growth rate value occurring at a lower value of $x / t$.

In figure 5 we also observe that as the thickness of the shear layer decreases the value of $g_{i}^{\max }$ increases. In fact $g_{i}^{\max }$ scales like $\delta_{1}^{-1}$ in this problem, and this can be seen as $g_{i}^{\text {max }} \approx 0.25$ for $\delta_{1}=\delta_{2}=0.5$ while $g_{i}^{\text {max }} \approx 0.5$ for $\delta_{1}=\delta_{2}=0.25$. The values of $g_{i}$ for the $\delta_{1}=0.25$ result cannot be calculated for $x / t \approx 0.5$ and 1.0 because close to these values the saddle point in the $\alpha$-plane has moved to a large value of $\alpha_{r}$, as discussed in $\S 2.2$. Thus the value of this saddle becomes difficult to calculate numerically because of numerical inaccuracies that occur in calculating the roots of the dispersion relation. This problem arises for thin shear layers and small values of $q$ and could be overcome by evaluating the integral in (2.6) numerically and calculating the growth rate from this. However, it can be noted by studying the $\alpha$-plane that in these limits no $s_{2}$ saddles become traversed by the integration contour; therefore it can be deduced that $g_{i} \rightarrow 0$ as $x / t \rightarrow 0.5$ and 1.0 as for the $\delta_{1}=0.5$ case, so no difficult numerical integrals need actually be evaluated. It is found in $\S 4$ that these parts of the growth rate are not required for calculating the breakup length for this value of $q$, so the calculation of these tails is irrelevant.

In figure $6(a)$ we plot $g_{i}$ as a function of $x / t$ for sinuous modes with the different density ratios $(0.5,0.5,1 / 500,0),(0.5,0.5,1 / 10,0)$ and $(0.5,0.5,1,0)$ numbered $1-3$ respectively. The most striking difference between the results displayed in figure $6(a)$ is that for $q=1$ (result 3 ) there is growth over a much larger range of $x / t$ characteristics. This is significant because now there is the chance that there could be growth along 

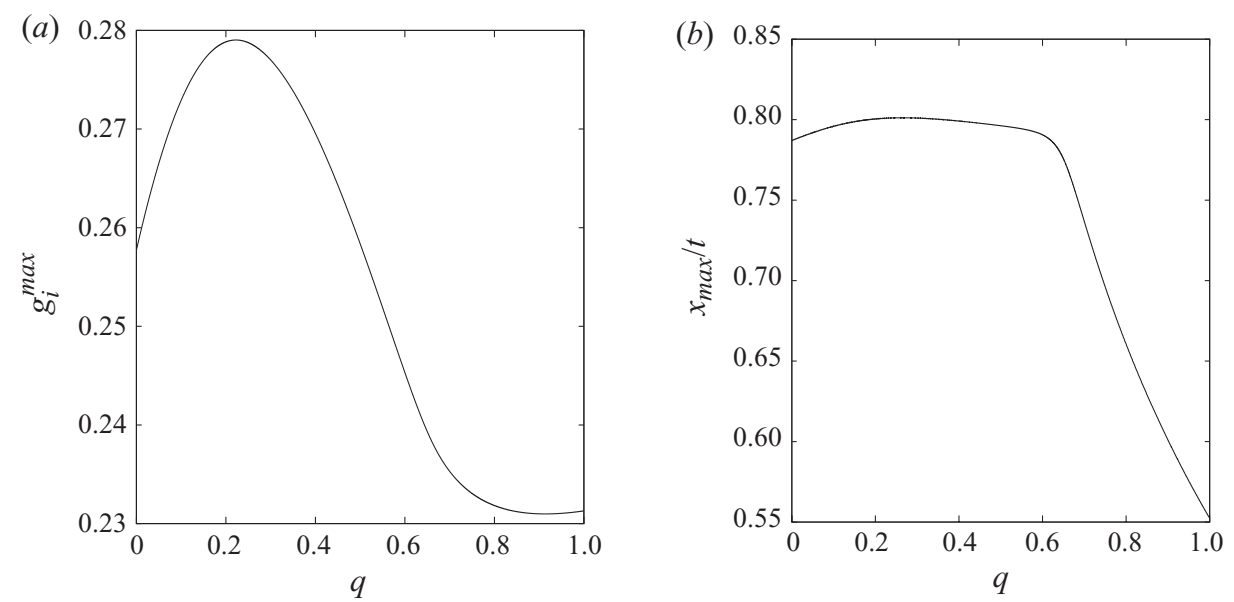

Figure 7. Plot of $(a)$ the maximum growth rate $g_{i}^{\max }$ as a function of $q$ for the case $\delta_{1}=\delta_{2}=0.5$ and $W=0$, and $(b)$ the value of $x / t$ at the maximum value.

the $x / t=0$ characteristic for particular parameter values; i.e. there could now exist an absolute instability. In this case there is a very weak absolute instability for $q=1$. As $q$ is increased from $1 / 500$ to 1 the maximum value of the growth rate, $g_{i}^{\max }$, initially increases up to $q \approx 1 / 5$ before decreasing as $q \rightarrow 1$. This can be seen in figure 7(a) for $\delta_{1}=\delta_{2}=0.5$. Figure $7(b)$ shows that the corresponding $x / t$ value increases slightly up to $q \approx 1 / 4$ before also reducing as $q \rightarrow 1$. This shows that as the density difference between the two fluids becomes closer to unity, breakup will occur at a later time for the same velocity profile because of the smaller growth rate, but also the disturbance will move slower along the jet. Weaker growth rates tend to delay breakup, but slower axial propagation velocities tend to move breakup towards the nozzle.

Figure $6(b)$ on the other hand shows the effect on the growth rate of varying the thickness of the outer shear layer, $\delta_{2}$, when $q=1 / 500$. The results show that thinning the shear layer in the less dense fluid increases the maximum growth rate by a small amount, and thickening the shear layer reduces the maximum growth rate by an even smaller amount. This small difference as $\delta_{2}$ is varied demonstrates that a thickening of the outer shear layer alone cannot be responsible for the increased breakup length observed in axisymmetric diesel jets (see the discussion in Sazhin et al. 2008). As $q$ increases, the effect of the outer shear layer increases but does not really become significant until $q \gtrsim 1 / 10$.

In figure $6(a)$ we find that for $q \leqslant 1$ the smallest value of $x / t$ for which growth is observed is $x / t=1 / 2=\beta$. Therefore we can generate results with $g_{i}>0$ for $x / t<1 / 2$ by considering a fixed shear layer thickness and by varying the magnitude of the jet velocity at the fluid interface within the shear layer. This is achieved by varying the parameter $\beta$ and allowing the parameters $\delta_{1}$ and $\delta_{2}$ to vary as

$$
\delta_{1}=a(1-\beta), \quad \delta_{2}=a \beta,
$$

where $a$ determines the thickness of the shear layer. Figure 8(a) shows that reducing $\beta$ from 0.6 (result 1) to 0.5 (result 2) with $a=1$ increases the range of $x / t$ characteristics along which there is growth. In fact the smallest value of $x / t$ for which there is growth is exactly $\beta$, which is verified by each of the four cases shown. We also note that the maximum value of $g_{i}$ varies only slightly as $\beta$ is varied, but the $x / t$ value at the maximum moves to smaller values as $\beta$ decreases. This increase in the range of $x / t$ 

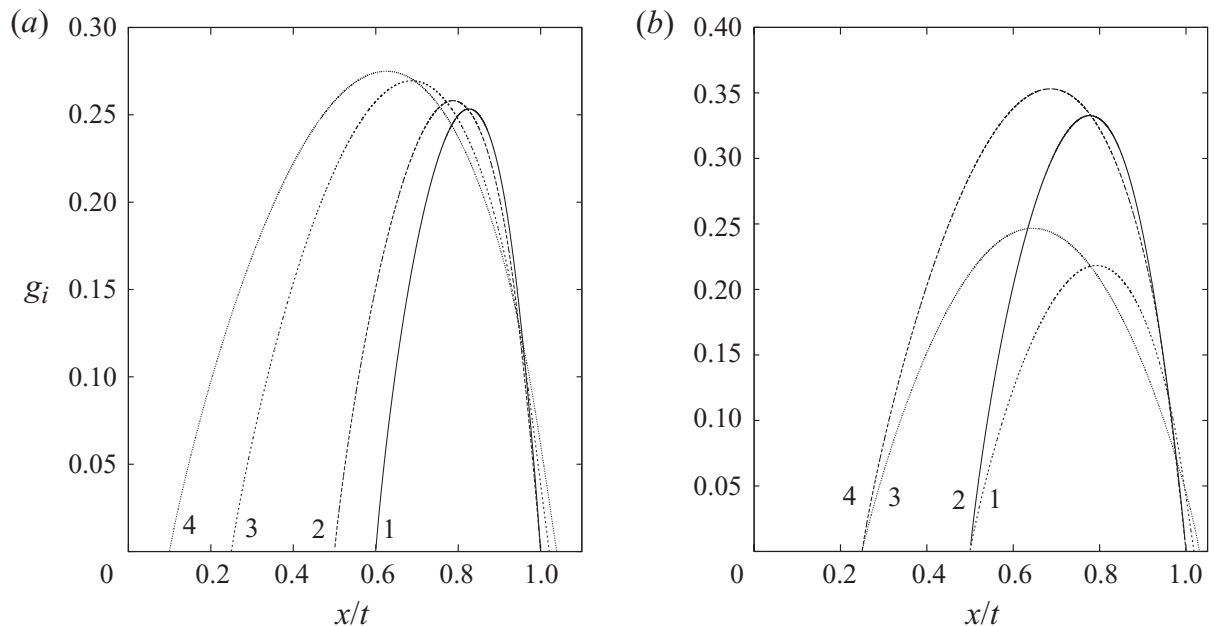

FIGURE 8. Plot of $g_{i}$ as a function of $x / t$ for sinuous modes of the velocity profile (3.1) with $q=1 / 500$ and $(a) a=1$ with $\beta=0.6,0.5,0.25$ and 0.1 , numbered $1-4$ respectively, and (b) $(a, \beta)=(1.25,0.5),(0.75,0.5),(1.25,0.25)$ and $(0.75,0.25)$, numbered $1-4$ respectively.
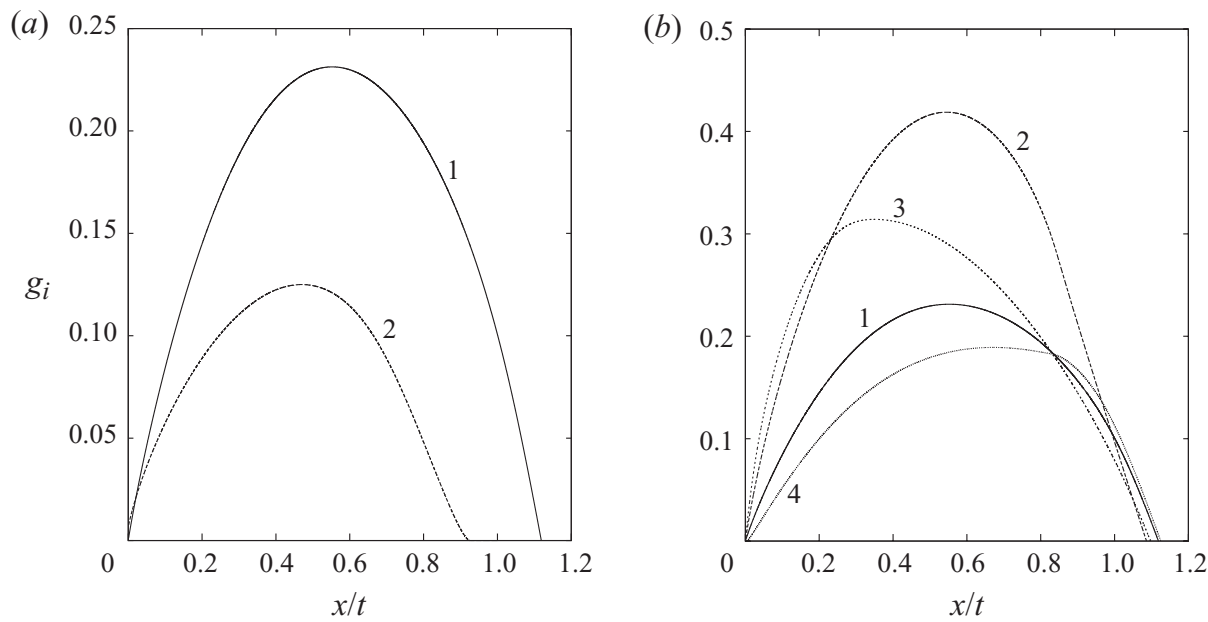

Figure 9. Plot of $g_{i}$ as a function of $x / t$ for $(a)(0.5,0.5,1,0)$ for sinuous and varicose modes, numbered 1 and 2 respectively, and $(b)$ for sinuous modes with $(0.5,0.5,1,0),(0.25,0.25,1,0)$, $(0.5,0.3,1,0)$ and $(0.5,0.7,1,0)$, numbered $1-4$ respectively.

values with $\beta$ is also seen for the varicose mode (not shown), which means that there is the possibility for an absolute instability, primarily in the varicose mode, as $\beta$ is reduced. This is examined further in figure 12. We should also note that for $\beta=0.25$ and 0.1 , characteristics with $x / t>1$ now have a positive growth rate. The implication of such solutions is discussed below. Figure $8(b)$ shows that different values of $a$ give the same effect as $\beta$ is varied, except that the overall growth rate is reduced as $a$ is increased and vice versa.

When we consider two fluids of equal density $(q=1)$ we find that varying parameters such as $\delta_{2}$ have a greater effect on the growth rate than they did for small values of $q(=1 / 500)$. Figure $9(a)$ plots both the sinuous (result 1) and varicose (result 2) modes for the case $(0.5,0.5,1,0)$, and these results compare to results 1 and 2 for the 

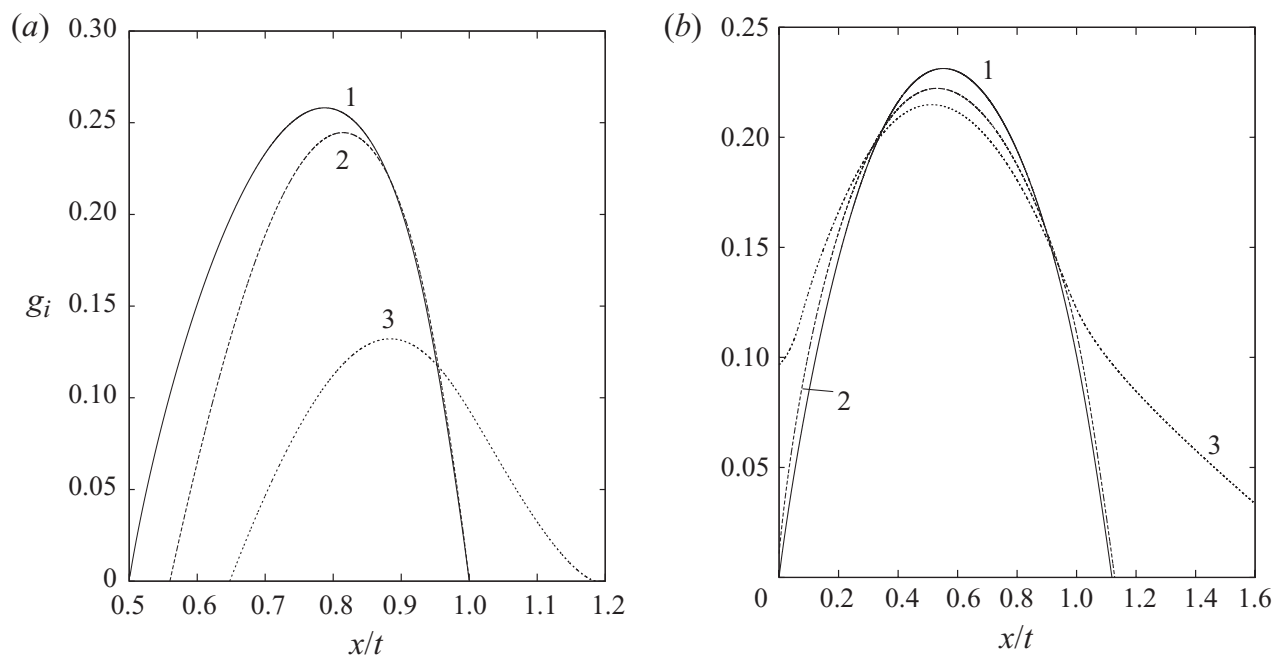

Figure 10. Plot of the sinuous mode growth rate $g_{i}$ as a function of $x / t$ for $\left(\delta_{1}, \delta_{2}, q, W\right)=$ (a) $(0.5,0.5,1 / 500,0),(0.5,0.5,1 / 500,0.001)$ and $(0.5,0.5,1 / 500,0.01)$, numbered $1-3$ respectively, and $(b)(0.5,0.5,1,0),(0.5,0.5,1,0.01)$ and $(0.5,0.5,1,0.02)$, numbered $1-3$ respectively.

$q=1 / 500$ case in figure 5 . For the $q=1$ results we see that there is a much larger difference between the maximum values of the growth rates of the two modes, with the sinuous mode growing more than twice as fast as the varicose mode at its maximum value. We also observe that the range of $x / t$ characteristics where the varicose mode is larger than the sinuous mode is greatly reduced for $q=1$ and is concentrated to a small region near $x / t=0$. In fact for this parameter set the varicose mode gives an absolute instability (i.e. $g_{i}>0$ at $x / t=0$ ). The sinuous mode on the other hand has growth along characteristics which can propagate at a group velocity greater than the speed of the jet $(x / t>1)$, although the value of $g_{i}$ on these characteristics is much less than the maximum value of $g_{i}$. These characteristic values would not be significant for a steady jet, as the jet is assumed to fill the whole domain $x \in[0, \infty)$. However for an accelerating jet emanating from the nozzle, the growth along these characteristics could prove important, because these wave packets would propagate along the jet, hit the front of the jet and then reflect back, setting up interference with other downstream-travelling wave packets which could induce breakup.

So far we have focused on jets with zero surface tension; however surface tension can have a major effect on both the convective and absolute instability properties of the jet. In figure $10(a)$ we consider the growth rate $g_{i}$ for sinuous modes as a function of $x / t$ for the cases $(0.5,0.5,1 / 500,0),(0.5,0.5,1 / 500,0.001)$ and $(0.5,0.5,1 / 500,0.01)$, numbered 1-3 respectively. Here we see that increasing the effect of surface tension decreases the maximum value of the growth rate; i.e. it has a stabilizing effect on the convective instability. This is because the surface tension forces act to suppress the instability waves on the surface of the fluid, thus reducing the ability of the free surface to break up. It is also interesting to note that for this small value of $q$, the value of $x / t$ at which the maximum value of $g_{i}$ occurs increases as $W$ is increased. In figure $10(b)$ we consider the effect of surface tension in the case $q=1$, i.e. when the two fluids have equal densities, again for the case $\delta_{1}=\delta_{2}=0.5$. Here we find that because $q$ is larger than in figure $10(a)$ we require larger values of $W$ to see a significant change in the growth rate $g_{i}$; thus we examine $W=0,0.01$ and 0.02 , which 

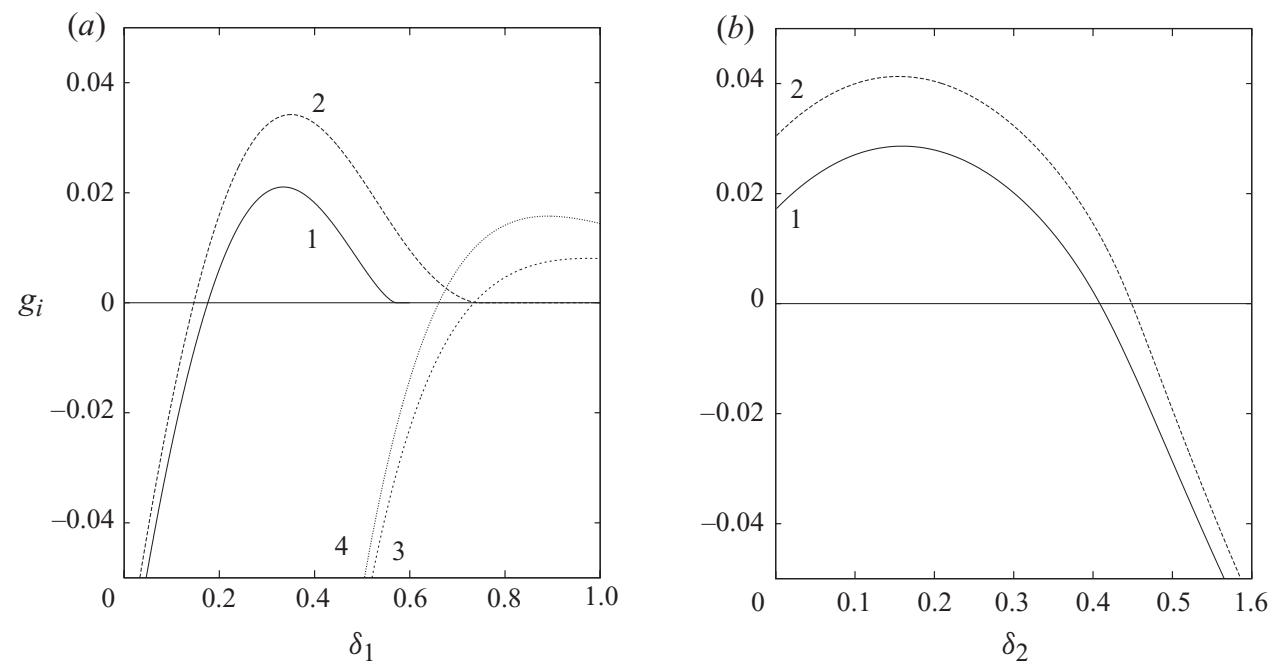

FIGURE 11. Plot of $g_{i}$ for varicose modes at the $s_{1}$ saddle on the inversion contour when $x / t=0$. In $(a) \delta_{1}=\delta_{2}$ and $(q, W)=(1,0),(1,0.01),(1 / 2,0)$ and $(1 / 2,0.01)$, numbered 1-4 respectively. In $(b)$ we fix $\delta_{1}=0.3$ and examine the value of $g_{i}$ at the $s_{1}$ saddle as a function of $\delta_{2}$ for $(1,0)$ and $(1,0.01)$, numbered 1 and 2 respectively. When this value is greater than 0 there is an absolute instability in the jet.

are numbered 1-3 respectively. As for $q=1 / 500$ in figure $10(a)$, the maximum value of $g_{i}$ reduces as $W$ is increased, but for $q=1$ the $x / t$ characteristic of the maximum growth rate moves to a smaller value. We also note that although surface tension has a stabilizing effect on the convective instability, it has a destabilizing effect on the absolute instability, as result 3 in figure $10(b)$ shows a weak absolute instability, while results 1 and 2 have no absolute instability. The destabilizing effect of surface tension for absolute instabilities has been studied by Lin \& Lian (1989), and we look at its effect on our jet profiles in figures 11 and 12 .

The major part of this study up to now has focused on the sinuous modes of the jet, but these modes are more stable than the varicose modes when it comes to absolute instabilities (Juniper 2006). Therefore, for the remainder of this section we focus our attention on varicose modes and investigate the conditions when they produce absolute instabilities in the jet. These are very important in steady jets, as they tell us when there will be disturbance growth at the nozzle which eventually leads to disintegration of the whole jet and the formation of a spray. We expect an absolute instability to disintegrate the jet in this fashion because we expect similar behaviour between this flow and the wake flow of Chomaz, Huerre \& Redekopp (1987); i.e. the jet is most unstable near the nozzle, so an absolute instability at the nozzle would generate an unstable global instability in the jet. Also, any vortex rings which occur, such as in the experiments of Monkewitz \& Sohn (1988), would be highly unstable to secondary instabilities at the high Reynolds numbers considered in this study, so breakup of the jet at the nozzle would be expected.

In figure 11 we plot the value of $g_{i}$ at the $s_{1}$ saddle on the inversion contour for various parameter values, and where this quantity is greater than zero we have an absolute instability in the flow. For result 1 , which has $\delta_{1}=\delta_{2}, W=0$ and $q=1$, we find that we have an absolute instability for $0.175<\delta_{1}<0.571$. This result extends the work of Juniper (2007) who suggests that a shear layer in the jet is not hugely 

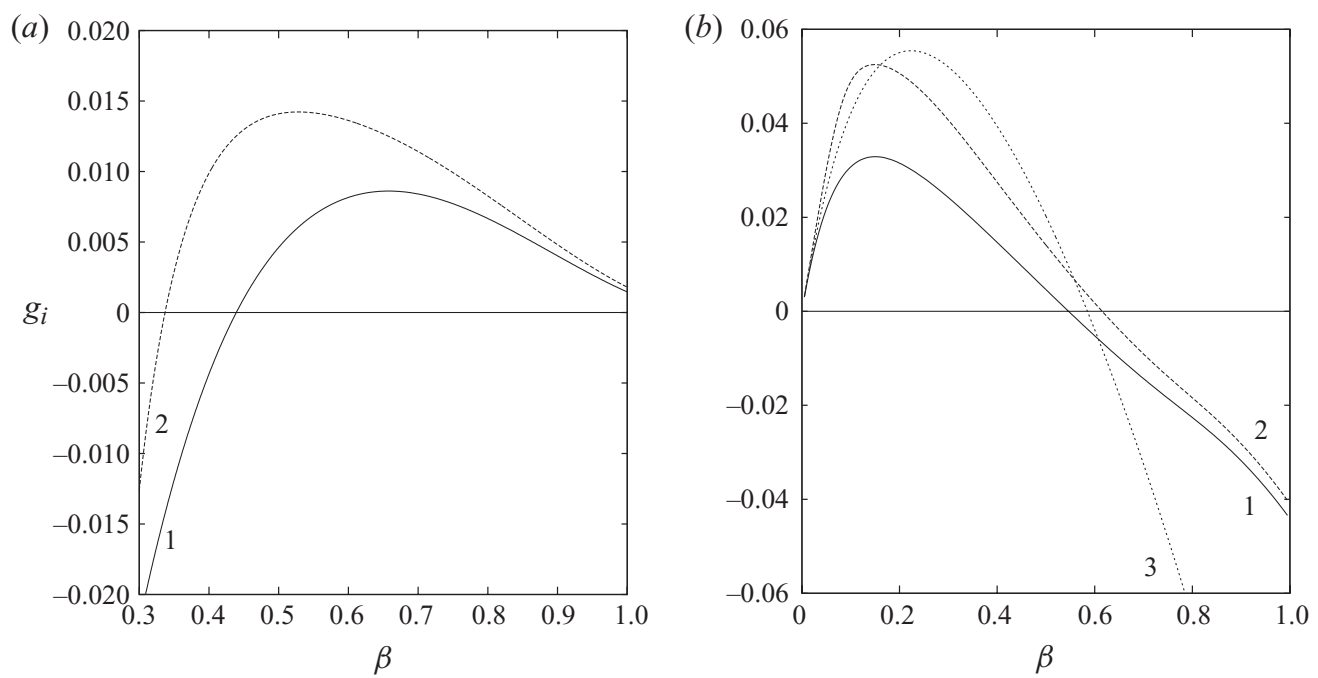

FIGURE 12. Plot of the value of $g_{i}$ for the varicose mode at the $s_{1}$ saddle on the inversion contour when $x / t=0$. For $(a)$ the velocity profile is fixed by the parameters (3.1) with $a=8 / 5$, $q=1 / 2$ and $W=0$ and 0.01 , numbered 1 and 2 respectively, while for $(b) \delta_{1}=\delta_{2}=0.8$ and $(q, W)=(1 / 2,0),(1 / 2,0.01)$ and $(1 / 10,0)$, numbered $1-3$ respectively. The value of $g_{i}$ is plotted as a function of $\beta$, and an absolute instability develops when $g_{i}$ is greater than 0 .

significant for its absolute stability properties; therefore he considered only the $\delta_{1}=0$ case which gives no absolute instability for the parameter range considered here. However figure 11(a) clearly shows that increasing the thickness of the shear layer will produce absolute instability, and when the shear layer becomes too wide the absolute instability stabilizes again. Result 2 shows that increasing the surface tension ( $W=0.01$ ) increases the range of values of $\delta_{1}$ for which there is an absolute instability to $0.146<\delta_{1}<0.743$. It also increases the magnitude of the absolute instability as discussed in Lin \& Lian (1989) and earlier in this section. Results 3 and 4 show the same absolute instability phenomena except with $q=1 / 2$. In this case the absolute instability occurs for a larger $\delta_{1}$ value and even extends to $\delta_{1}=1$ where the profile becomes a triangular jet. Note that this result is the opposite of that observed by Srinivasan et al. (2010) who found that for low-density jets $(q>1)$ the absolute instability occurs as $\delta_{1} \rightarrow 0$ and becomes convectively unstable as $\delta_{1}$ increases.

In figure $11(b)$ we fix $\delta_{1}=0.3$ with $q=1$ and investigate how varying the outer shear layer $\delta_{2}$ affects the absolute instability. From figure 11(a) we know that $\delta_{2}=0.3$ for this parameter set leads to an absolute instability, but figure 11(b) shows that reducing the thickness of the outer shear layer increases the absolute instability to $\delta_{2}=0.160$, and then the absolute instability reduces as $\delta_{2} \rightarrow 0$, still giving an absolute instability at $\delta_{2}=0$. Increasing $\delta_{2}$ from 0.3 just reduces the absolute instability, and at $\delta_{2}=0.408$ the absolute instability stabilizes. Result 2 shows the destabilizing effect of $W=0.01$. For the results in figure 11 we found that reducing $q$ much below $1 / 2$ removed the absolute instability, so for dense jets the flow is only convectively unstable.

In figure $12(a)$ we examine the absolute instability properties of the fixed shear layer profile (3.1) with $a=8 / 5$ where we vary the velocity at the fluid interface, effectively varying the position of the fluid interface relative to a fixed shear layer, for the density ratio $q=1 / 2$. We plot the value of $g_{i}$ at the $s_{1}$ saddle, which lies on the inversion contour, as a function of $\beta$. This profile was chosen because from figure 11(a) we 
know that this profile, with $\beta=1 / 2$, has an absolute instability with $W=0$. We see that increasing $\beta$ enhances the absolute instability up to $\beta=0.658$, while reducing $\beta$ only stabilizes the absolute instability, and when $\beta=0.440$ the absolute instability has vanished, and the flow is only convectively unstable. Here, setting $W=0.01$ (result 2) does increase the range of $\beta$ for which there is an absolute instability, but only slightly, and it also shifts the position of the maximum absolute instability to a smaller value of $\beta$. Figure $12(b)$ shows that absolute instabilities in jets with $q<1$ tend to occur only for values of $\beta \lesssim 0.7$ when $\delta_{1}=\delta_{2}=0.8$. Therefore for the CFD profiles from figure 2(a) that we consider in $\S 4$, we may not encounter an absolute instability. However this study is still significant for other jets where $\beta$ might be less than 0.7 because of the physical properties of the fluids being considered.

We have now extended the study of the stability properties of a steady planar jet and identified some new phenomena associated with the inclusion of the shear layer at the jet edge. In the next section we use these results to help explain the experimental jet breakup results seen in figure 1.

\section{Breakup length calculations}

In this section we use the stability results from $\S 3$ to estimate breakup lengths of liquid jets. Experiments, such as those by Hiroyasu et al. (1982), show that a liquid jet injected from a nozzle has a breakup length which increases for small injection velocities, reaches a maximum value and then levels off for large velocities; see figure 13 from their study or figure 1 of this study. The jets in these experiments were axisymmetric water jets injected into pressurized nitrogen, where the injection process was long enough to justify our assumption that the jets are essentially steady, and therefore our steady theory can be used to explain the results. The proposed model in this paper includes several undetermined parameters intended to model physical effects beyond the scope of the present study, such as the role of nonlinearity in breakup. In principle, improved quantitative agreement could be sought through empirically adjusting the parameters to fit the data better, but this is not the approach we have taken, nor is that the goal of this research; our intention is to obtain a qualitative understanding of the important physical processes. This limitation of our goal is partly related to the fact that Hiroyasu et al. (1982) considered axisymmetric jets, while our model was developed for planar jets.

We examine how the parameters used in $\S 3$ affect the jet breakup length by studying four different profiles. Before discussing the profiles we first need to relate the parameters $\delta_{1}, \delta_{2}$ and $\beta$ to the maximal velocity $V$ of the jet. By considering the CFD results in figure 2 we can estimate these parameters. By considering these parameters in figure 13 we can see that $\delta_{1}$ has the approximate form

$$
\delta_{1}=\delta_{\infty}+\delta^{\prime} V^{-1 / 2}
$$

to leading order, where $\delta^{\prime}+\delta_{\infty}$ is the non-dimensional thickness of the shear layer of the jet when $V=1$ and $\delta_{\infty}$ is the thickness of the shear layer in the limit $V \rightarrow \infty$. This result also agrees with the experimental results of Marmottant \& Villermaux (2004) who plot the velocity profiles of an air jet injected into static air and again find that the shear layer in the jet is proportional to $V^{-1 / 2}$. The variation of $\beta$ with $V$ is much smaller than for $\delta_{1}$; however it still increases with increasing $V$, and this variation has an effect on the breakup length calculations. We fix the parameter $\delta_{2}$ with respect to $V$, as this is approximately true for the CFD calculations. Note here that as $V$ is varied the parameters that depend upon $V$ are also varied, such as the surface tension effects, which appear as $W / V^{2}$ in the dispersion relation (2.4). 


$\begin{array}{cccc}\begin{array}{c}\text { Maximum velocity } \\ \left(\mathrm{m} \mathrm{s}^{-1}\right)\end{array} & \beta & \delta_{1} & \delta_{2} \\ 44 & 0.48 & 0.85 & 0.27 \\ 81 & 0.49 & 0.77 & 0.26 \\ 182 & 0.56 & 0.60 & 0.24 \\ 340 & 0.63 & 0.44 & 0.23\end{array}$

TABLE 1. The approximate values of $\delta_{1}, \delta_{2}$ and $\beta$ for the CFD simulations shown in figure $2(a)$.

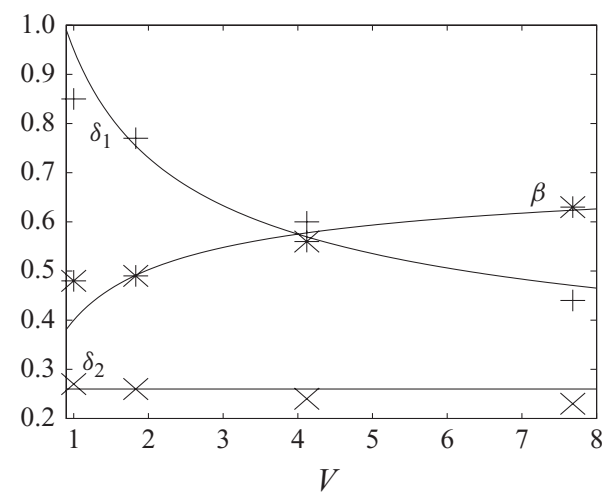

FIGURE 13. Plot of the normalized data from table 1 with the profile 1 approximations.

The four profiles we examine in this section are the following:

profile 1: $\quad \delta_{1}=0.2+0.75 \mathrm{~V}^{-1 / 2}, \quad \delta_{2}=0.26, \quad \beta=0.75-0.35 \mathrm{~V}^{-1 / 2} ;$

profile 2: $\quad \delta_{1}=\delta_{2}=0.2+0.75 V^{-1 / 2}, \quad \beta=0.5$;

profile 3: $\quad \delta_{1}=0.2+0.75 \mathrm{~V}^{-1 / 2}, \quad \delta_{2}=0.26, \quad \beta=0.5$;

profile $4: \quad \delta_{1}=0.8(1-\beta), \quad \delta_{2}=0.8 \beta, \quad \beta=0.75-0.35 \mathrm{~V}^{-1 / 2}$.

Profile 1 gives a profile with the parameters $\delta_{1}, \delta_{2}$ and $\beta$ which fit the table 1 data. The values in table 1 are estimated at the dotted fluid interface in figure 2 , and then profile 1 is generated by fitting curves through the data, as shown in figure 13. Note that we have non-dimensionalized the data in table 1 by the velocity of the slowest jet. A different non-dimensionalization would have led to different functions $\delta_{1}, \delta_{2}$ and $\beta$. Profiles 2-4 pull out particular characteristics from profile 1 so that their individual effects on the breakup length can be investigated. Profile 2 fixes the value of $\beta$ and allows both $\delta_{1}$ and $\delta_{2}$ to thin with $V$, highlighting the effect of reducing $\delta_{1}$ in the small $q$ limit in which the effect of $\delta_{2}$ is small (see figure $6 b$ ). Profile 3 is like profile 2 , but now we fix $\delta_{2}=0.26$ so that comparisons with profile 2 will show how variations in $\delta_{2}$ affect the breakup length for $q$ close to unity, where this effect is significant. Also this profile shows the effect of fixing $\beta$ when compared with profile 1 . Profile 4 allows us to investigate the effect of allowing $\beta$ to increase on the breakup length by fixing the shear layer profile and increasing $\beta$ with $V$. One point to note is that profiles $1-4$ have been chosen to approximately coincide in the $V \rightarrow \infty$ limit, and plots of each profile at $V=1,5$ and 10 can be seen in figure 14 .

The actual breakup length calculation is not a stability problem; it is a transition problem, where we wish to calculate the position at which the initial disturbance from 

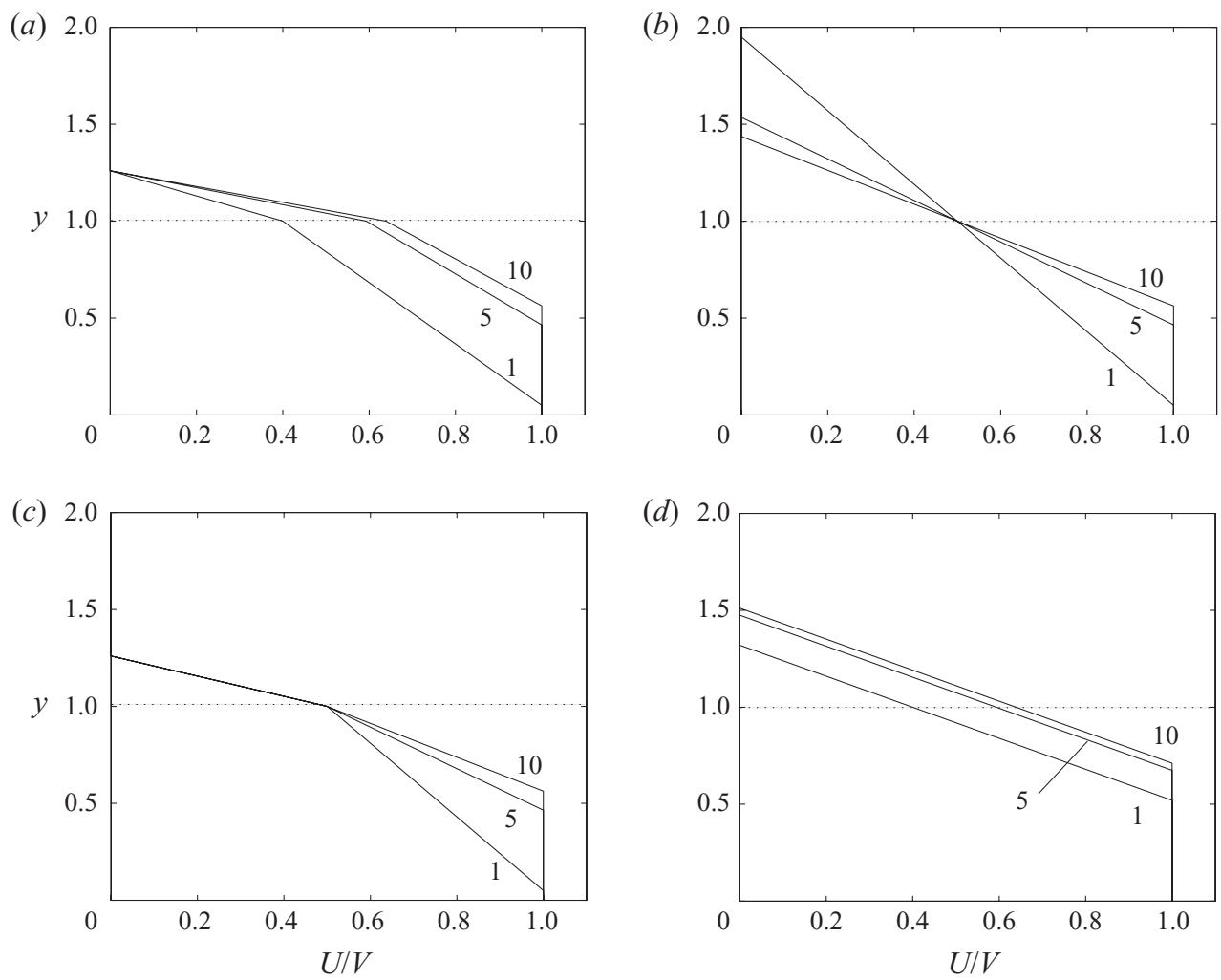

Figure 14. Plot of velocity profiles $1-4$ in $(a)-(d)$ respectively, at $V=1,5$ and 10 . The dotted line indicates the fluid interface.

the nozzle has reached some threshold amplitude at which the disturbance can no longer be assumed to be a linear perturbation to the basic jet velocity and nonlinear effects occur. We are concerned with calculating the disturbance amplitude relative to a range of basic flow velocities, so we expect that the quantity $|\hat{v}|_{\max } /|V|$ will be relevant in estimating the position at which nonlinearity starts to dominate, and we assume further that this will be a precursor to rapid jet breakup. The subscript $\max$ above means maximizing over the $y$-direction. This ratio was studied by Shen (1961) who used it in unsteady flows to determine whether or not the flow was instantaneously stable or unstable. Therefore we calculate when the ratio $|\hat{v}|_{\text {max }} /|V|$ reaches some critical value, and we say that at this point breakup has occurred. It is equally possible to consider the ratio of the streamwise disturbance velocity and the basic velocity $|\hat{u}|_{\max } /|V|$ or the ratio of the disturbance kinetic energy to the base flow kinetic energy, but this would just correspond to choosing a different critical threshold value. The threshold amplitude is unknown; we have chosen $\left|\hat{v}_{b}\right|_{\text {max }} /|V|=40 \tilde{v}_{0}$ in this paper, where $\left|\hat{v}_{b}\right|_{\max }$ is the disturbance amplitude that triggers jet breakup and $\tilde{v}_{0}=\left|v_{0}\right|_{\max } /|V|$ is the initial disturbance amplitude ratio. Thus we assume that the initial amplitude of the eigenfunction grows linearly with $|V|$. It may turn out that this is not the case, and in general $\left|v_{0}\right|_{\max }=f(|V|)$ for some function $f$; however there is no experimental evidence to determine this function, and therefore, fixing $\left|v_{0}\right|_{\text {max }} /|V|$ reduces the number of parameters we have to consider, as well as being a sensible assumption, as the initial disturbance amplitude is likely to increase with jet velocity. A 40-fold amplification of the eigenmode may not be the correct value for breakup, 


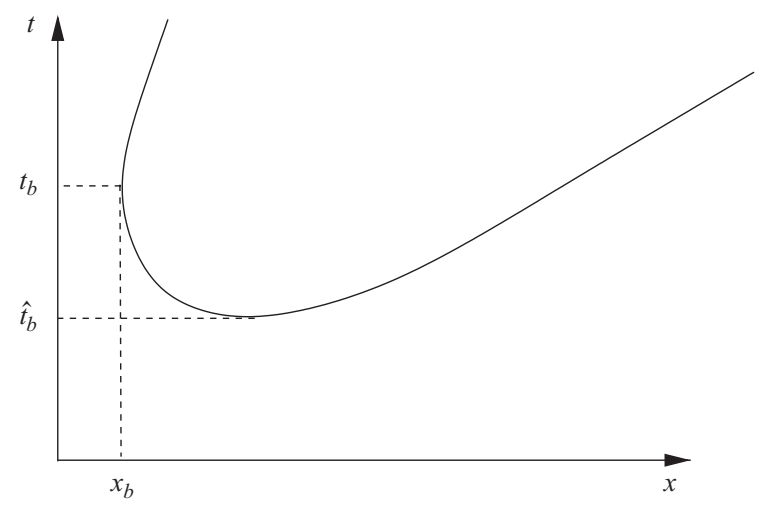

FIGURE 15. Schematic diagram of the $x-t$ plane, showing the definition of the breakup length $x_{b}$, the corresponding breakup time $t_{b}$ and the initial breakup time $\hat{t}_{b}$.

but changing this value will have only a quantitative effect on the results presented in this section, and the overall trend of the results will be the same. Typically the amplification factor is a function of $q$, but we fix it in this study, as we are seeking only qualitative agreement with experiments.

Under these assumptions, the ratio of the initial disturbance amplitude, $\left|v_{0}\right|_{\max }$, to the amplitude of the jet at breakup along the characteristic $x / t=C=$ constant is found from the method of steepest descent to be the solution of

$$
\frac{\left|\hat{v}_{b}\right|_{\max }}{|V|}=\frac{\left|v_{0}\right|_{\max }}{|V|\left[t_{b}(C)\left|\partial^{2} g / \partial \alpha^{2}\right|_{x / t=C}\right]^{1 / 2}} \exp \left(g_{i}(C) t_{b}(C)\right),
$$

where $t_{b}(C)$ is the time of breakup along the particular characteristic $C$ and $v_{0}(y)$ is the corresponding initial disturbance eigenfunction at the saddle point corresponding to $x / t=C$, whose magnitude can be adjusted. Then for a steady jet the breakup length along the characteristic $x / t=C$ is given by the following formula:

$$
x_{b}^{s, v}(C)=\left.\frac{\partial \omega}{\partial \alpha}\right|_{x / t=C} t_{b}^{s, v}(C)=C t_{b}^{s, v}(C),
$$

where $\partial \omega / \partial \alpha$ evaluated at the dominant saddle point on $x / t=C$ is equal to $C$. The superscript $s$ or $v$ signifies whether this breakup length is for the sinuous or varicose mode. The actual breakup length $x_{b}$ is then taken to be the $\min \left(x_{b}^{s}, x_{b}^{v}\right)$ over every characteristic $x / t=C$ with $g_{i}(C)>0$. A schematic diagram of the $x-t$ plane, defining $x_{b}$ and $t_{b}$, is given in figure 15 .

In figure $16(a)$ we see that $x_{b}(V)$ for $q=1 / 500$ (result 1 ) shows an increase in value for small $V$ before decreasing as $V$ continues to increase. In these results the breakup length is dominated by the sinuous modes of the jet. As $q$ is increased to $q=1 / 10$ (result 2) we see a similar structure to that for $q=1 / 500$, except now the overall breakup length is reduced. When these results are compared with the experimental results in figure 1 (or figure 13 of Hiroyasu et al. 1982), we see qualitative agreement. The results in Hiroyasu et al. (1982) were plotted for various pressures $P_{a}$ of the gas into which the fluid is injected. Increasing $P_{a}$ corresponds to increasing $q$, and the results in figure 13 of Hiroyasu et al. (1982) correspond to $q=1 / 1000,1 / 100,1 / 30$ and $1 / 25$ from top to bottom respectively. In figure $16(a)$, the $q=1 / 2$ result shows a slightly different behaviour, with $x_{b}(V)$ reducing and levelling off as $V$ increases. The main reason for this is that because the parameters $\delta_{1}, \delta_{2}$ and $\beta$ are actually functions 

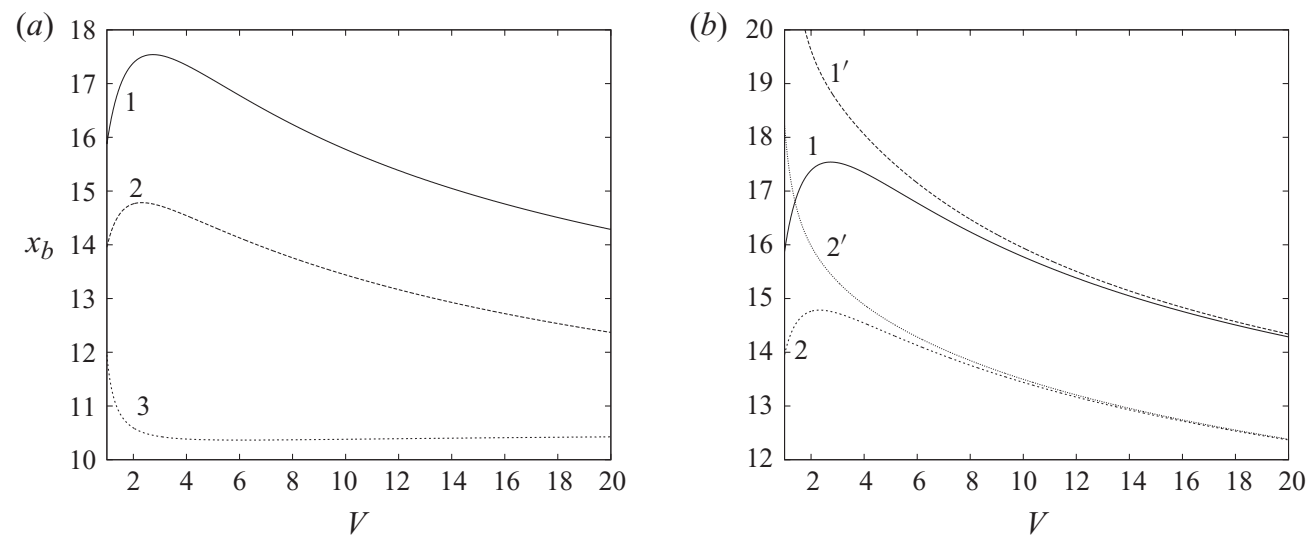

Figure 16. (a) Plot of $x_{b}(V)$ for $(q, W)=(1 / 500,0),(1 / 10,0)$ and $(1 / 2,0)$, numbered $1-3$ respectively, all for profile 1 . $(b)$ Replotting of results 1 and 2 of $(a)$, with the primes denoting the corresponding results with $W=0.01$.
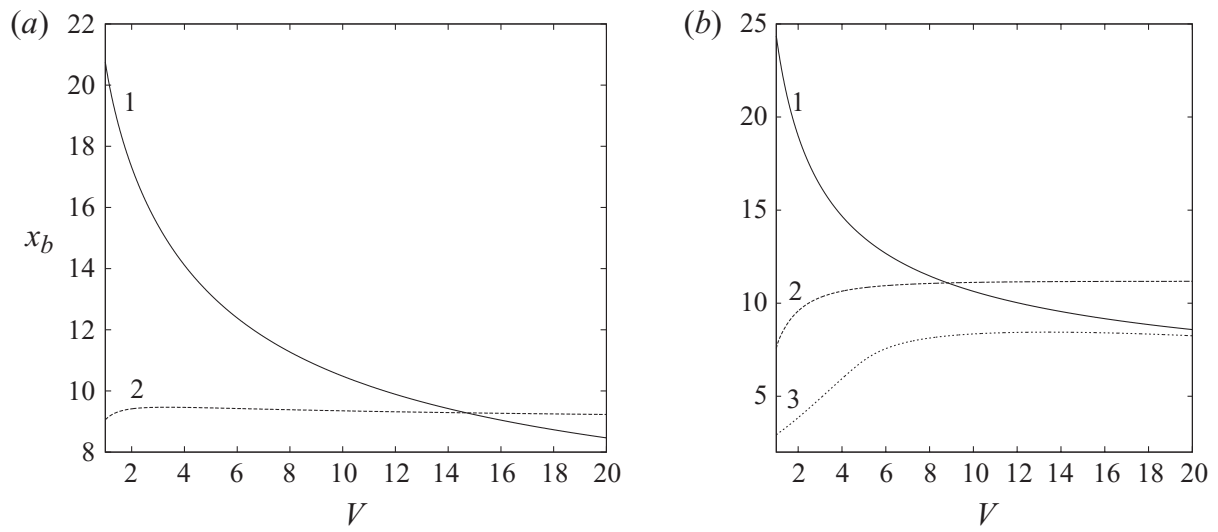

Figure 17. (a) Plot of $x_{b}(V)$ for profiles 2 and 4 , numbered 1 and 2 respectively, for $(q, W)=$ $(1 / 500,0)$. (b) Plot of $x_{b}(V)$ for profiles 2,4 and 3 for $(1 / 2,0)$, numbered $1-3$ respectively.

of $q$ as well as $V$, a jet with $q=1 / 2$ may have a different profile from that given by profile 1 . However we have ignored this extra complication in this study for simplicity.

Figure $16(b)$ replots the $q=1 / 500$ and $1 / 10$ results of figure $16(a)$ and includes the corresponding results with $W=0.01$ denoted by primes. For both results the inclusion of surface tension stabilizes the flow and causes the breakup length to increase; also the increase in $x_{b}$ for small $V$ is removed. Note that as $V$ is increased the effect of surface tension decreases, and the $W \neq 0$ results tend to the $W=0$ results rapidly, owing to surface tension occurring as $W / V^{2}$ in the dispersion relation (2.4). The surface tension from the experiments of Hiroyasu et al. (1982) is $O\left(10^{-4}\right)$, so the surface tensions we consider here are larger than in the experiments.

In figure 17 we plot $x_{b}(V)$ for profiles 2-4 with $(q, W)=(a)(1 / 500,0)$ and (b) $(1 / 2,0)$. In figure $17(a)$ the results for profile 3 are not plotted, as they cannot be distinguished from the profile 2 results for this value of $q$. This confirms the unimportance of the value of $\delta_{2}$ at this particular density ratio. For curve 1 in figure $17(a)$, we already know that thinning the shear layer $\delta_{1}$ causes the maximum growth 

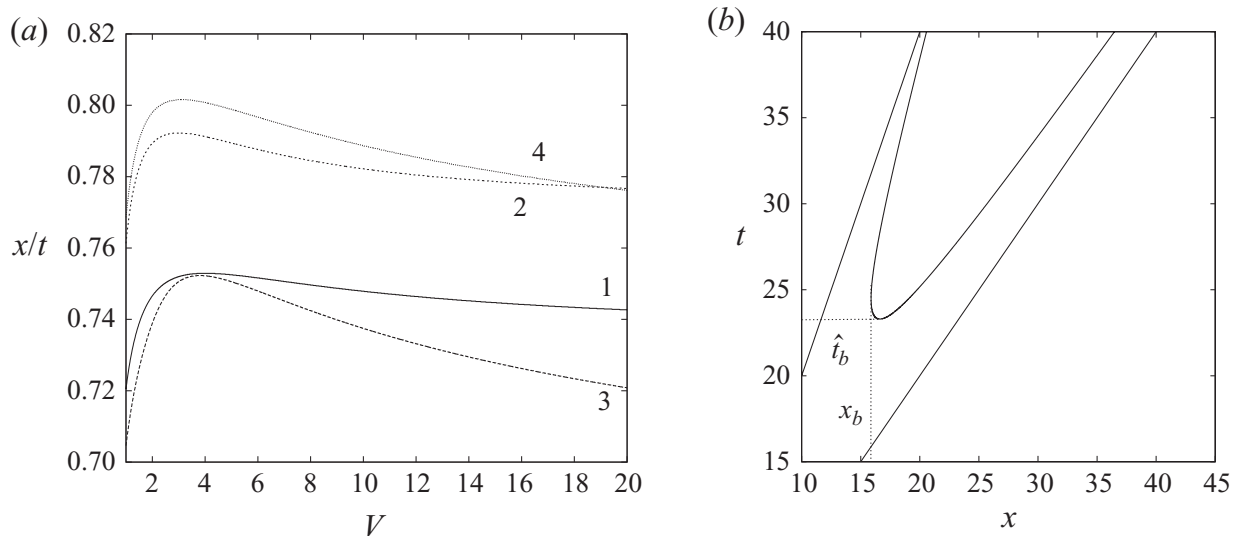

Figure 18. (a) Plot of the value of $x / t$ along which breakup occurs for the sinuous mode of profile 2 for $(1 / 500,0)$ and $(1 / 2,0)$, numbered 1 and 3 respectively. Results 2 and 4 give the corresponding characteristics for the maximum value of the growth rate. $(b)$ Plot of the $x-t$ plane for the sinuous mode of result 1 of figure $16(a)$ at $V=1$. The curve gives the contour along which $|\hat{v}| /|V|=\left|\hat{v}_{b}\right| /|V|$, while the solid straight lines give the edges of the wave packet. The dotted lines give the values of $x_{b}$ and the initial time of breakup, $\hat{t}_{b}$.

rate to increase (see figure 5), which itself causes the breakup length to reduce as a function of $V$. For this value of $q$, the value of $x / t$ along which breakup occurs is within $5 \%$ of the characteristic along which $g_{i}$ is maximum (see figure $18 a$ ). However, increasing the fluid velocity at the fluid interface (profile 4, curve 2) in figure 17( $a$ ) causes the breakup length to increase slightly at small values of $V$ before remaining approximately constant over the rest of the range. This is due to the maximum growth rate reducing as $\beta$ increases (see figure 8). In figure $17(b)$ for $q=1 / 2$ we see similar results for profile 2 (curve 1) and profile 4 (curve 2), but now breakup occurs along characteristics further away from the characteristic along which $g_{i}$ is maximum with approximately $8 \%$ difference in characteristic values (see figure $18 a$ ). Therefore we cannot just examine how the maximum growth rate varies with $V$ to give an indication of how $x_{b}$ will vary. These differences can also be seen in the $x-t$ plot of figure $18(b)$ for the $q=1 / 500$ result from figure 16(a). Here we plot the contour at the edges of the wave packet and the contour where $|\hat{v}| /|V|=\left|\hat{v}_{b}\right| /|V|$ to show where breakup occurs. We can see the clear difference between the breakup lengths given by $x_{b}$ and the position at which breakup first occurs. In figure $17(b)$ we plot $x_{b}$ for profile 3 and see that unlike for $q=1 / 500$ the results are qualitatively different from those of profile 2 . Here there is an increase in $x_{b}$ initially before levelling off for larger velocities; therefore at this density ratio the shear layer in the lighter fluid is significant.

In this section we have shown qualitative agreement between our theoretical predictions and the experimental results of Hiroyasu et al. (1982), including a possible explanation of the turning point behaviour in $x_{b}(V)$ that can arise at some parameter combinations. We have demonstrated that the turning point can occur even when $W=0$, so this behaviour is not a surface tension effect. However the current results need to be extended to axisymmetric jets to fully confirm this.

\section{Conclusions and discussion}

In this paper we have used a spatio-temporal stability analysis to examine the linear stability of a steady two-dimensional planar liquid jet which has a shear layer 
in both the inner and outer fluids. Using a piecewise linear velocity profile we showed that for two fluids with a density ratio $q=1 / 500$ (which corresponds approximately to cold diesel fuel being injected into compressed air at 5 atmospheres) the important characteristic of the velocity profile was the size of the shear layer in the denser fluid, as this had the largest effect on the growth rate of disturbances. The width of the shear layer in the less dense fluid on the other hand made no significant contribution at this value of $q$. However as the density ratio of the two fluids was increased the width of the second shear layer contributed more significantly to the stability properties of the jet and consequently to the breakup length properties. Surface tension was found to stabilize the convective stability properties of the jet by reducing the maximum growth rate value for a given velocity profile.

We investigated the effect of thickening the liquid shear layer on the absolute instability of the jet and found that the finite shear layer produces an absolute instability in jets with smaller values of the density parameter than was found in the work of Juniper (2006) who considered only infinitely thin shear layers. Juniper (2006) found that absolute instability occurs for $q \gtrsim 1.25$, while we have shown that an absolute instability occurs for at least $q>0.5$. Therefore, the structure of the shear layer is important in these types of problems. The work in the present paper also found that increasing the fluid velocity at the fluid interface within the shear layer of the jet can destabilize the absolute instability, but ultimately the flow is convectively unstable for the values of $\beta$ observed in our CFD calculations (where $\beta$ is the ratio of the fluid velocity at the interface to the maximum velocity). In the case of absolute instabilities, surface tension was found to act as a destabilizing effect in agreement with the work of Lin \& Lian (1989).

Using the spatio-temporal analysis we have been able to demonstrate that the breakup length of a jet reduces as the shear layer in the jet thins, while the breakup length increases as the magnitude of the fluid velocity at the fluid interface increases. By combining these two effects into a profile which describes more accurately what occurs in a real jet, we have been able to produce qualitative agreement with the experiments of Hiroyasu et al. (1982).

We note that in the axisymmetric jet case, surface tension has a destabilizing role at small jet velocities because it can trigger the pinch-off of drops of radius comparable to the radius of the jet, so this may also act to reduce breakup lengths at small jet velocities, but this may not be an important mechanism at parameters relevant to fuel injection. When extending this work to axisymmetric jets we also have to be aware that the wave with zero azimuthal wavenumber behaves like the varicose mode of the planar jet case and is destabilized by surface tension, so some differences in the the results may be observed (Juniper 2008). This however is beyond the scope of the present paper.

The authors would like to thank Cyril Crua for supplying the experimental data for the CFD simulations. This work is supported by the EPSRC under grants EP/F069855/1, EP/G000034/1 and EP/F058276/1. We would also like to thank the anonymous referees whose comments led to an improved version of this paper.

\section{Appendix. The dispersion relation}

The dispersion relation in $\S 2$ has the form

$$
\hat{c}_{4} \omega^{4}+\hat{c}_{3} \omega^{3}+\hat{c}_{2} \omega^{2}+\hat{c}_{1} \omega+\hat{c}_{0}=0,
$$


408 M. R. Turner, J. J. Healey, S. S. Sazhin and R. Piazzesi

where

$$
\begin{aligned}
\hat{c}_{4}= & -c_{3}, \\
\hat{c}_{3}= & \beta \alpha V c_{3}-c_{2}, \\
\hat{c}_{2}= & \beta \alpha V c_{2}-c_{1}-T_{3}\left(T_{1}^{2}-1\right)\left(1+T_{2}\right)\left(T_{3}-1\right) W \alpha^{4}, \\
\hat{c}_{1}= & \beta \alpha V c_{1}-c_{0}-\left[\frac{\beta V}{\delta_{2}} T_{3}\left(T_{2}-T_{3}\right)\left(T_{1}^{2}-1\right)\right. \\
& \left.+\left(1+T_{2}\right)\left(T_{3}-1\right)\left(\frac{(1-\beta) V T_{1}^{2}}{\delta_{1}}-T_{3}\left(\alpha V\left(T_{1}^{2}-1\right)+\frac{(1-\beta) V T_{1}}{\delta_{1}}\right)\right)\right] W \alpha^{4}, \\
\hat{c}_{0}= & \beta \alpha V c_{0}-\frac{\beta V}{\delta_{2}}\left(T_{2}-T_{3}\right)\left[\frac{(1-\beta) V T_{1}^{2}}{\delta_{1}}-T_{3}\left(\alpha V\left(T_{1}^{2}-1\right)+\frac{(1-\beta) V T_{1}}{\delta_{1}}\right)\right] W \alpha^{4},
\end{aligned}
$$

with

$$
\begin{aligned}
& c_{3}=-\left(T_{1}^{2}-1\right)\left(1+T_{2}\right)\left(T_{3}-1\right)\left(q T_{3}+1\right), \\
& c_{2}=\left(1+T_{2}\right)\left(q T_{3}+1\right)\left(T_{3}-1\right)\left((1+\beta) \alpha V\left(T_{1}^{2}-1\right)+\frac{(1-\beta) V T_{1}}{\delta_{1}}\right) \\
& +\frac{\beta V}{\delta_{2}}\left(T_{1}^{2}-1\right)\left(T_{3}(1-q)-T_{2}\left(1-q T_{3}^{2}\right)\right)+\frac{(1-\beta) V T_{1}^{2}}{\delta_{1}}\left(1+T_{2}\right)\left(T_{3}+q\right)\left(1-T_{3}\right) \\
& +V\left(\frac{(1-\beta)}{\delta_{1}}-\frac{\beta q}{\delta_{2}}\right)\left(T_{1}^{2}-1\right)\left(1+T_{2}\right) T_{3}\left(T_{3}-1\right), \\
& c_{1}=\beta \alpha V\left[\left(1+T_{2}\right)\left(q T_{3}+1\right)\left(1-T_{3}\right)\left(\alpha V\left(T_{1}^{2}-1\right)+\frac{(1-\beta) V T_{1}}{\delta_{1}}\right)\right. \\
& \left.+\frac{\beta V}{\delta_{2}}\left(T_{1}^{2}-1\right)\left(T_{2}\left(1-q T_{3}^{2}\right)-T_{3}(1-q)\right)+\frac{(1-\beta) V T_{1}^{2}}{\delta_{1}}\left(1+T_{2}\right)\left(T_{3}-1\right)\left(T_{3}+q\right)\right] \\
& +\frac{\beta V}{\delta_{2}}\left(\alpha V\left(T_{1}^{2}-1\right)+\frac{(1-\beta) V T_{1}}{\delta_{1}}\right)\left(T_{2}\left(1-q T_{3}^{2}\right)-T_{3}(1-q)\right) \\
& +\frac{\beta(1-\beta) V^{2} T_{1}^{2}}{\delta_{1} \delta_{2}}\left(T_{3}^{2}-q-T_{2} T_{3}(1-q)\right) \\
& +V\left(\frac{(1-\beta)}{\delta_{1}}-\frac{\beta q}{\delta_{2}}\right)\left[\left(1+T_{2}\right) T_{3}\left(1-T_{3}\right)\left(\alpha V\left(T_{1}^{2}-1\right)+\frac{(1-\beta) V T_{1}}{\delta_{1}}\right)\right. \\
& \left.+\frac{\beta V T_{3}}{\delta_{2}}\left(T_{1}^{2}-1\right)\left(T_{2}-T_{3}\right)+\frac{(1-\beta) V T_{1}^{2}}{\delta_{1}}\left(1+T_{2}\right)\left(T_{3}-1\right)\right], \\
& c_{0}=\beta \alpha V\left[\frac{\beta V}{\delta_{2}}\left(\alpha V\left(T_{1}^{2}-1\right)+\frac{(1-\beta) V T_{1}}{\delta_{1}}\right)\left(T_{3}(1-q)-T_{2}\left(1-q T_{3}^{2}\right)\right)\right. \\
& \left.+\frac{\beta(1-\beta) V^{2} T_{1}^{2}}{\delta_{1} \delta_{2}}\left(T_{2} T_{3}(1-q)-T_{3}^{2}+q\right)\right] \\
& +\frac{\beta V^{2}}{\delta_{2}}\left(\frac{(1-\beta)}{\delta_{1}}-\frac{\beta q}{\delta_{2}}\right)\left(T_{3}-T_{2}\right)\left(T_{3}\left(\alpha V\left(T_{1}^{2}-1\right)+\frac{(1-\beta) V T_{1}}{\delta_{1}}\right)\right. \\
& \left.-\frac{(1-\beta) V T_{1}^{2}}{\delta_{1}}\right)
\end{aligned}
$$


for varicose modes, and

$$
\begin{aligned}
\hat{c}_{4}= & -c_{3}, \\
\hat{c}_{3}= & \beta \alpha V c_{3}-c_{2}, \\
\hat{c}_{2}= & \beta \alpha V c_{2}-c_{1}-\left(T_{1}^{2}-1\right)\left(1+T_{2}\right)\left(T_{3}-1\right) W \alpha^{4}, \\
\hat{c}_{1}= & \beta \alpha V c_{1}-c_{0}+\left[\frac{\beta V}{\delta_{2}}\left(T_{2}-T_{3}\right)\left(T_{1}^{2}-1\right)\right. \\
& \left.-\left(1+T_{2}\right)\left(T_{3}-1\right)\left(\alpha V\left(T_{1}^{2}-1\right)-\frac{(1-\beta) V}{\delta_{1}}\left(T_{1}-T_{3}\right)\right)\right] W \alpha^{4}, \\
\hat{c}_{0}= & \beta \alpha V c_{0}+\frac{\beta V}{\delta_{2}}\left(T_{3}-T_{2}\right)\left[\alpha V\left(T_{1}^{2}-1\right)-\frac{(1-\beta) V}{\delta_{1}}\left(T_{1}-T_{3}\right)\right] W \alpha^{4},
\end{aligned}
$$

with

$$
\begin{aligned}
& c_{3}=\left(T_{1}^{2}-1\right)\left(1+T_{2}\right)\left(T_{3}-1\right)\left(T_{3}+q\right), \\
& c_{2}=\left(1+T_{2}\right)\left(T_{3}+q\right)\left(1-T_{3}\right)\left((1+\beta) \alpha V\left(T_{1}^{2}-1\right)-\frac{(1-\beta) V T_{1}}{\delta_{1}}\right) \\
& +\frac{\beta V}{\delta_{2}}\left(T_{1}^{2}-1\right)\left(T_{2} T_{3}(1-q)-T_{3}^{2}+q\right)+\frac{(1-\beta) V}{\delta_{1}}\left(1+T_{2}\right)\left(q T_{3}+1\right)\left(1-T_{3}\right) \\
& +V\left(\frac{(1-\beta)}{\delta_{1}}-\frac{\beta q}{\delta_{2}}\right)\left(T_{1}^{2}-1\right)\left(1+T_{2}\right)\left(1-T_{3}\right), \\
& c_{1}=\beta \alpha V\left[\left(1+T_{2}\right)\left(T_{3}+q\right)\left(T_{3}-1\right)\left(\alpha V\left(T_{1}^{2}-1\right)-\frac{(1-\beta) V T_{1}}{\delta_{1}}\right)\right. \\
& \left.+\frac{\beta V}{\delta_{2}}\left(T_{1}^{2}-1\right)\left(T_{3}^{2}-q-T_{2} T_{3}(1-q)\right)+\frac{(1-\beta) V}{\delta_{1}}\left(1+T_{2}\right)\left(T_{3}-1\right)\left(q T_{3}+1\right)\right] \\
& +\frac{\beta V}{\delta_{2}}\left(\alpha V\left(T_{1}^{2}-1\right)-\frac{(1-\beta) V T_{1}}{\delta_{1}}\right)\left(T_{3}^{2}-q-T_{2} T_{3}(1-q)\right) \\
& +\frac{\beta(1-\beta) V^{2}}{\delta_{1} \delta_{2}}\left(T_{3}(1-q)+T_{2}\left(q T_{3}^{2}-1\right)\right) \\
& +V\left(\frac{(1-\beta)}{\delta_{1}}-\frac{\beta q}{\delta_{2}}\right)\left[\left(1+T_{2}\right)\left(T_{3}-1\right)\left(\alpha V\left(T_{1}^{2}-1\right)-\frac{(1-\beta) V T_{1}}{\delta_{1}}\right)\right. \\
& \left.+\frac{\beta V}{\delta_{2}}\left(T_{1}^{2}-1\right)\left(T_{3}-T_{2}\right)+\frac{(1-\beta) V T_{3}}{\delta_{1}}\left(1+T_{2}\right)\left(T_{3}-1\right)\right], \\
& c_{0}=\beta \alpha V\left[\frac{\beta V}{\delta_{2}}\left(\alpha V\left(T_{1}^{2}-1\right)-\frac{(1-\beta) V T_{1}}{\delta_{1}}\right)\left(T_{2} T_{3}(1-q)-T_{3}^{2}+q\right)\right. \\
& \left.+\frac{\beta(1-\beta) V^{2}}{\delta_{1} \delta_{2}}\left(T_{2}\left(1-q T_{3}^{2}\right)-T_{3}(1-q)\right)\right] \\
& +\frac{\beta V^{2}}{\delta_{2}}\left(\frac{(1-\beta)}{\delta_{1}}-\frac{\beta q}{\delta_{2}}\right)\left(T_{2}-T_{3}\right)\left(\alpha V\left(T_{1}^{2}-1\right)-\frac{(1-\beta) V}{\delta_{1}}\left(T_{1}-T_{3}\right)\right)
\end{aligned}
$$

for sinuous modes, where $T_{1}=\tanh \left(\alpha\left(L-\delta_{1}\right)\right), T_{2}=\tanh \left(\alpha\left(L+\delta_{2}\right)\right), T_{3}=\tanh (\alpha L)$ and $q=\rho_{2} / \rho_{1}$. 


\section{REFERENCES}

Arcoumanis, C., Gavaises, M., Flora, H. \& Roth, H. 2001 Visualisation of cavitation in diesel engine injectors. Mec. Ind. 2, 375-381.

BAtchelor, G. K. \& Gill, A. E. 1962 Analysis of the stability of axisymmetric jets. J. Fluid Mech. 14, 529-551.

BriggS, R. J. 1964 Electron-Stream Interaction with Plasmas. MIT Press.

Chomaz, J. M., Huerre, P. \& Redekopp, L. G. 1987 Models of hydrodynamic resonances in separated shear flows. In Procceedings of 6th Symposium on Turbulent Shear Flows, Toulouse, France (ed. M. Hoffmeister), pp. 321-326. Springer.

Crua, C. 2002 Combustion processes in a diesel engine. PhD Thesis, University of Brighton, Brighton, UK.

Domann, R. \& Hardalupas, Y. 2004 Breakup model for accelerating liquid jets. In Proceedings of 42nd AIAA Aerospace Science Meeting and Exhibition, Reno, Nevada. AIAA Paper 2004-1155.

Drazin, P. G. \& ReID, W. H. 1981 Hydrodynamic Stability. Cambridge University Press.

Eggers, J. \& Villermaux, E. 2008 Physics of liquid jets. Rep. Prog. Phys. 71, 036601-1-79.

EsCH, R. E. 1957 The instability of a shear layer between two parallel streams. J. Fluid Mech 3, 289-303.

Ferziger, J. H. \& Peric, F. M. 2004 Computational Methods for Fluid Dynamics, 3rd edn. Springer.

Funada, T., JosePh, D. D. \& Yamashita, S. 2004 Stability of a liquid jet into incompressible gases and liquids. Intl J. Multiphase Flow 30, 1279-1310.

Hagerty, W. W. \& Shea, J. F. 1955 A study of the stability of plane fluid sheets. J. Appl. Mech. 22, 509-514.

Hashimoto, H. \& Suzuki, T. 1991 Experimental and theoretical study of fine interfacial waves on thin liquid sheet. JSME Intl J. II 34 (3), 277-283.

HEALEY, J. J. 2006 A new convective instability of the rotating-disk boundary layer with growth normal to the disk. J. Fluid Mech. 560, 279-310.

Healey, J. J. 2007 Enhancing the absolute instability of a boundary layer by adding a far-away plate. J. Fluid Mech. 579, 29-61.

Healey, J. J. 2009 Destabilizing effects of confinement on homogeneous mixing layers. J. Fluid Mech. 623, 241-271.

Heurre, P. \& Monkewitz, P. A. 1990 Local and global instabilities in spatially developing flows. Annu. Rev. Fluid Mech. 22, 473-537.

Heywood, J. B. 1998 Internal Combustion Engine Fundamentals. McGraw-Hill.

Hinch, E. J. 1991 Perturbation Methods. Cambridge University Press.

Hiroyasu, H., Shimizu, M. \& Arai, M. 1982 The break-up of high speed jet in a high pressure gaseous atmosphere. In Proceedings of the 2nd International Conference on Liquid Atomization and Spray Systems, pp. 69-74.

Huerre, P. 2000 Open shear flow instabilities. In Perspectives in Fluid Dynamics: A Collective Introduction to Current Research (ed. G. K. Batchelor, H. K. Moffat \& M. G. Worster), pp. 159-230. Cambridge University Press.

JUNIPER, M. P. 2006 The effect of confinement on the stability of two-dimensional shear flows. J. Fluid Mech. 565, 171-195.

JUNIPER, M. P. 2007 The full impulse response of two-dimensional jet/wake flows and implications for confinement. J. Fluid Mech. 590, 163-185.

JUNIPER, M. P. 2008 The effect of confinement on the stability of non-swirling round jet/wake flows. J. Fluid Mech. 605, 227-252.

KARIMI, K. 2007 Characterisation of multiple-injection diesel sprays at elevated pressures and temperatures. PhD Thesis, University of Brighton, Brighton, UK.

Lesshafft, L. \& Huerre, P. 2007 Linear impulse response in hot round jets. Phys. Fluids 19, 024102.

LI, X. \& TANKIN, R. R. 1991 On the temporal stability of a two-dimensional viscous liquid sheet. J. Fluid Mech 226, 425-443.

Lin, S. P. \& Lian, Z. W. 1989 Absolute instability of a liquid jet in a gas. Phys. Fluids 1 (3), 490-493.

Lin, S. P., Lian, Z. W. \& Creighton, B. J. 1990 Absolute and convective instability of a liquid sheet. J. Fluid Mech 220, 673-689.

Marmottant, P. \& Villermaux, E. 2004 On spray formation. J. Fluid Mech. 498, 73-111.

Monkewitz, P. A. \& Sohn, K. D. 1988 Absolute instability in hot jets. AIAA J. 26, 911-916. 
RaYleigh, LORD 1894 The Theory of Sound, 2nd edn. Macmillian.

Raynal, L., Harion, J-L., Favre-Marinet, M. \& Binder, G. 1996 The oscillatory instability of plane variable-density jets. Phys. Fluids 8, 993-1006.

Rees, S. J. \& Juniper, M. P. 2009 The effect of surface tension on the stability of unconfined and confined planar jets and wakes. J. Fluid Mech. 633, 71-97.

ReEs, S. J. \& Juniper, M. P. 2010 The effect of confinement on the stability of viscous planar jets and wakes. J. Fluid Mech. 656, 309-336.

Sazhin, S. S., Crua, C., Kennaird, D. A. \& Heikal, M. R. 2003 The initial stage of fuel spray penetration. Fuel 82 (8), 875-885.

Sazhin, S. S., Martynov, S. B., Kristyadi, T., Crua, C. \& Heikal, M. R. 2008 Diesel fuel spray penetration, heating, evaporation and ignition: modelling vs. experimentation. Intl J. Engng Syst. Model. Simul. 1 (1), 1-19.

Schmid, P. J. \& Henningson, D. S. 2001 Stability and Transition in Shear Flows. Springer.

SHEN, S. F. 1961 Some considerations on the laminar stability of time-dependent basic flows. J. Aerosp. Sci. 28, 397-404, 417.

SöDERBERG, L. D. 2003 Absolute and convective instability of a relaxational plane liquid jet. J. Fluid Mech. 493, 89-119.

SÖDERBERG, L. D. \& Alfredsson, P. H. 1998 Experimental and theoretical stability investigations of plane liquid jets. Eur. J. Mech B/Fluids 17, 689-737.

Sreenivasan, K. R., Raghu, S. \& Kyle, D. 1989 Absolute instability in variable density round jets. Exp. Fluids 7, 309-317.

Srinivasan, V., Hallberg, M. P. \& Strykowski, P. J. 2010 Viscous linear stability of axisymmetric low-density jets: parameters influencing absolute instability. Phys. Fluids 22, 024103.

Stone, R. 1992 Introduction to Internal Combustion Engines. MacMillan.

Youngs, D. L. 1982 Time-dependent multi-material flow with large fluid distortion. In Numerical Methods for Fluid Dynamics (ed. K. W. Morton \& M. J. Baines), pp. 273-285. Academic Press.

Yu, M. H. \& Monkewitz, P. A. 1990 The effect of non-uniform density on the absolute instability of planar inertial jets and wakes. Phys. Fluids A 2 (7), 1175-1181. 